\title{
IUSTINIANOS - DER NEUE AUGUSTUS? ADOPTION, NAME UND PROPAGANDA EINES KÜNFTIGEN KAISERS*
}

\author{
JÁN BAKYTA
}

\begin{abstract}
Justinian - the New Augustus?

Adoption, Name and Propaganda of a Future Emperor

This is a reappraisal of the evidence for the adoption of the later emperor Justinian by his uncle Justin, which has been questioned by Noethlichs (2001). Even though Justinian's references to Justin as his 'father' are not conclusive, the epigram Anth. Gr. I, 97 can be evaluated as a reliable positive indication. The name 'Justinian' is the original name of the emperor and has nothing to do with the adoption, whereas the names 'Petrus' and 'Sabbatius' as attested in the consular diptychs are secondary names, the former perhaps being a model for the subsequent fashion of devotional cognomina. Finally, the related issues of possible propaganda by Justinian (his comparison to Augustus adopted by Caesar) and its reception (by Procopius or John the Lydian) are discussed.
\end{abstract}

Keywords: Justinian I; Justin I; Octavianus Augustus; Corippus; Procopius of Caesarea; John the Lydian; Roman onomastics; Roman polyonymy

Obwohl Iustinianos I. (527-565) möglicherweise sogar als „le plus célèbre de tous les empereurs byzantins" ${ }^{\text {"1 }}$ gelten kann, werden sich wohl kaum Forscher*innen finden, die zu behaupten bereit wären, dass wir genügend die Hintergründe, Verhältnisse oder Karriere dieses Mannes aus der Zeit kennen, ehe er hinreichend prominent wurde, um von den zeitgenössischen - jedoch zunächst spärlichen - Quellen registriert oder aber zumindest aus der rückwärtigen - also nicht unbedingt verlässlichen - Perspektive in den späteren Zeugnissen erwähnt zu werden. Diesen Zeitpunkt kann man zwar irgendwo während der Regierung seines Onkels Iustinos I. (518-527) ansetzen, doch gibt es offene Fragen auch für diese Periode, wie vor ein paar Jahren eine Arbeit von B. Croke gezeigt hat. Entgegen der gewohnten Sichtweise wird darin nämlich folgende weitgehend neue

* Der Artikel entstand im Rahmen des Projekts der Karlsuniversität „Univerzitní centrum pro studium antické a středověké myšlenkové tradice“ (UNCE 204002/2012). Ich danke den anonymen Gutachter*innen für ihre Bewertungen des Beitrags sowie einige sprachlichen Korrekturen und meinem Kollegen Reiner Lipp (Karlsuniversität, Prag) für die durchlaufende Revision der Sprache des Textes.

1 So Stein (1949: 222). 
Rahmeninterpretation geboten: „Justinian's authority during the reign of Justin ... was not abrupt and absolute, but grudging and gradual." 2

Der australische Forscher ist dabei auch auf die Problematik der Adoption des Iustinianos durch Iustinos eingegangen und hat sie klar präsentiert. ${ }^{3}$ Doch hat er die Ansicht außer Acht gelassen, dass die Historizität jener Adoption unsicher ist. Zweifel in diesem Sinne hat nämlich K. L. Noethlichs artikuliert ${ }^{4}$ und damit auch weitere Kenner der justinianischen Problematik beeinflusst ${ }^{5}$ - obwohl dies außerhalb der deutschsprachigen Wissenschaft, wie etwa der Fall Crokes zeigt, der Aufmerksamkeit entgeht.

Im Folgenden möchte ich diese Frage wieder aufgreifen und die Historizität der Adoption untermauern sowie den in der Forschung eher vernachlässigten Namen des Iustinianos - auch eins der Argumente - besprechen. Schließlich werde ich einige Überlegungen zur Propaganda und Rezeption dieser Adoption vortragen, die zwar jeweils unsicher bleiben müssen, trotzdem aber hoffentlich zur Fachdiskussion beitragen können in einer Zeit, da sich die Erforschung der Ideologien, der Diskurse, des Denkens und der Propaganda auch in Konstantinopel des 6. Jahrhunderts immer größerer Aufmerksamkeit erfreut.

Eines ist von vornherein klar: Da in den Quellen die fragliche Adoption nirgendwo direkt erwähnt wird, kann man sie nicht unmittelbar anhand der Evidenz beweisen, und trotz der Plausibilität der zu erbringenden Argumentation kann sie folglich angesichts der notwendigen indirekten Beweisführung nicht als völlig gesichert gelten. In dieser Hinsicht gebührt Noethlichs' Leistung somit volle Anerkennung.

Was die indirekten Hinweise betrifft, führt Croke zweierlei auf, und zwar den Namen des Adoptierten und die Verweise in den Quellen auf Iustinos als Iustinianos' Vater.6 Noethlichs nennt zusätzlich als drittes ein von Croke in die zweite Kategorie der Belege eingereihtes Epigramm der Anthologia Graeca (bzw. Palatina). ${ }^{7}$ Dann widmet er sich aber nur der Vater-Sohn-Terminologie (also der zweiten Gruppe der Belege; unklar ist, ob implizit auch dem Epigramm), obwohl man doch erwarten sollte, dass er alle aufgezählten Argumente widerlegen würde, falls er die Hypothese der Adoption ernsthaft entkräften wollte. ${ }^{8}$

Alle Belege für die Bezeichnung des Iustinos als Iustinianos' Vater werden aus der Perspektive bzw. als die Aussage des Letztgenannten formuliert und stammen erst aus der Zeit seiner (Allein)herrschaft nach Iustinos' Tod. ${ }^{9}$ Noethlichs' Einwand gegen die übli-

Croke (2007: 56).

Croke (2007: 20-21).

Noethlichs (2001: 674-675).

5 So Meier (2003a: 187 mit Anm. 398): „die Frage einer Adoption ist nach wie vor ungeklärt“ (allerdings nur mit Verweis auf Noethlichs) und Leppin (2011:358-359 Anm. 32): „Noethlichs ... äußert berechtigte Bedenken“ (im Anschluss mit dem Vorschlag einer Kompromisslösung auf der Grundlage des Namens des Iustinianos, der, wie unten gezeigt wird, dazu jedoch nicht geeignet ist).

6 Croke (2007: 20).

7 Noethlichs (2001: 674-675).

8 Es mag sein, dass Noethlichs die übrigen Belege für nichtig und deswegen keines Wortes wert gehalten hat. Doch scheint mir dieses Versäumnis eher durch das Bemühen um die Knappheit oder durch eine gewisse Flüchtigkeit der Arbeit an der Stelle bedingt zu sein (vgl. die falsche Zuschreibung der zitierten Arbeit nicht an Alan, sondern „Av.“ [= Averil] Cameron und die Angabe, dass darin die Adoption „,or der Kaisererhebung des I.“ [= Iustinianos; recte Iustinos] behauptet wird). Dadurch möchte ich aber die Qualitäten von Noethlichs' (2001) Arbeit als Ganzem nicht schmälern.

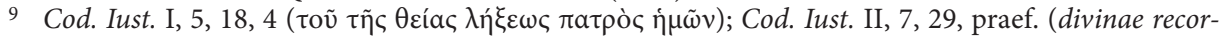
dationis Iustini patris nostri); Inst. Iust. II, 7, 3 (divus Iustinus pater noster); Inst. Iust. II, 12, 4 (divi

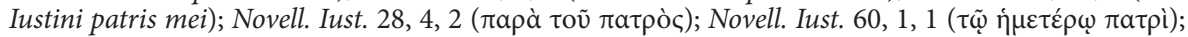

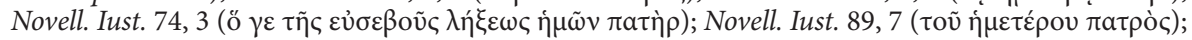


che Interpretation lautet folgendermaßen: „die Vater-Sohn-Terminologie läßt sich auch traditionell als Ausdruck der Hochachtung vor dem Vorgänger (u. Onkel) erklären. "10 Leider dokumentiert der Forscher einen solchen Gebrauch des Wortes $\pi \alpha \tau \eta ́ \rho$ bzw. pater nicht, der vor allem für die Kaiser (bzw. die Produkte der kaiserlichen Kanzlei im Namen des Herrschers) etwa des 5. und früheren 6. Jahrhunderts hätte eruiert werden müssen. ${ }^{11}$

In Corippus' Panegyrikus auf Iustinianos' Nachfolger Iustinos II. (565-578) findet man eine Stelle, die Noethlichs' Zweifel an der Aussagekraft des auf den Vorgänger eines Kaisers des 6. Jahrhunderts angewandten Vaterbegriffs zunächst als durchaus berechtigt erscheinen lässt. Auf den zweiten Blick sieht die Sachlage anders aus, doch ist diese Stelle in unseren Zusammenhängen immerhin lehrreich.

Sie lautet wie folgt: « Haec est illa dies », laeti dixere clientes, " quam pater ille bonus sancto praedixerat ore ». ${ }^{12}$ Es wird hier vom Tag der Thronbesteigung von Iustinos II. gesprochen, und der Vorhersagende soll der Forschung zufolge der verstorbene Iustinianos sein. ${ }^{13}$ Wird hier dieser als Iustinos' Vater bezeichnet? ${ }^{14}$ Genealogisch könnte er nur als sein Onkel angesprochen werden, und an eine Adoption kann man angesichts der sonst fehlenden Belege sowie der Umstände der Thronbesteigung von Iustinos II. kaum denken. ${ }^{15}$

Sollte man bei jener Erklärung bleiben, dann müsste man zugeben, dass es unter Iustinos II. möglich war, ohne nähere Begründung von Iustinianos als Vater des regierenden Kaisers zu sprechen; warum soll das dann nicht auch unter Iustinianos bei der Bezeichnung seines Vorgängers Iustinos I. möglich gewesen sein? Zu beachten wäre, dass die Bezeichnung dabei nicht (oder zumindest nicht primär) als Ausdruck der Hochachtung dienen sollte, sondern als ausgesprochen genealogischer Terminus; denn letzten Endes wird der Beleg aus der Perspektive der clientes, nicht des Kaisers formuliert.

Eine vielleicht bessere Lösung wäre es, „den Vater“ hier wirklich als eine Bezeichnung der Hochachtung aufzufassen. Aber auch in diesem Fall hätten wir mit der Perspektive anderer und nicht derjenigen des Nachfolgers des Verstorbenen zu tun.

Wie mir jedoch scheint, ist mit pater ille bonus überhaupt nicht Iustinianos gemeint. Denn nach anderen Quellen sollen sogar zwei heilige Männer Iustinos die Regierung prophezeit haben. ${ }^{16}$ Der Panegyriker kann sehr wohl auf einen von ihnen - oder auf eine ähnliche Geschichte von der Weissagung Iustinos' Kaisertums - angespielt haben.

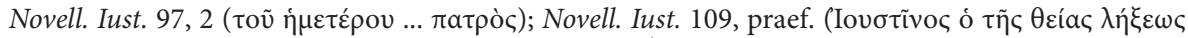

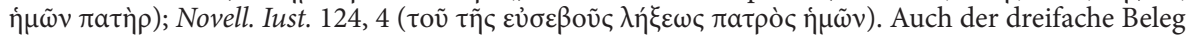
ACO IV/1: 186, Z. 37f. (piae recordationis patris eius), 39 und 41f. stellt eigentlich die Reproduktion Iustinianos' Äußerung dar.

10 Noethlichs (2001: 675).

11 Vgl. aber Anm. 22.

12 Coripp. Iust. II, 98-99. Die Übersetzung: „,Dies ist der Tag,〈 haben in Freude die Untertanen gesagt, >den jener gute Vater mit seinem heiligen Munde vorhergesagt hat."

13 Unmissverständlich Av. Cameron (1976: 157). So versteht die Stelle offensichtlich auch Whitby (1994: 87) sowie, seiner Übersetzung zufolge, Antès (1981:37).

14 Antès (1981: 37) und Whitby (1994: 87) sind offenbar dieser Meinung; Av. Cameron (1976: 157) berührt die Frage stricto sensu nicht, sondern postuliert nur die Gleichung pater ille bonus = Iustinianos und bleibt auch in der Übersetzung (Av. Cameron 1976: 96 [,the good father"]) wortgetreu und fügt nichts Weiteres hinzu.

15 Zur Genealogie s. PLRE III: 754 (s.v. Iustinus 5); zur Thronbesteigung vgl. z.B. die Zusammenfassungen von Rosen (2001b: 783-785) oder Leppin (2011: 334).

16 Eustrat. Vita Eutychii 1850-1867; Vita Symeonis Stylitae iunioris 202-203. In der Literatur wird diese Tatsache z.B. von Stein (1919: 26 Anm. 1), PLRE III: 755 (s.v. Iustinus 5) oder Rosen (2001b: 784) - 
Die Corippus-Stelle macht jedenfalls darauf aufmerksam, dass die Belege für Iustinos

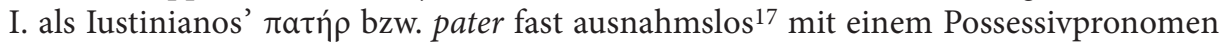
versehen sind (und somit deutlich aus der Perspektive des Kaisers formuliert werden). Der Gedanke, dass der Inhalt der so von Iustinianos seinem Vorgänger Iustinos zugeschriebenen Vaterrolle ihm gegenüber etwa nur das Greisenalter oder irgendeine Tugend war, die Hochachtung verdienen würden, wäre absurd. Das hat vermutlich auch Noethlichs gesehen, wenn er von der Hochachtung vor dem Vorgänger und (sekundär, wie es scheint) dem Onkel schreibt.

Allerdings würde dann Iustinianos von seinen Vorgängern nur Iustinos auf diese Weise ehren, was nicht besonders glaubhaft ist. ${ }^{18}$ Bemerkenswert ist die Aussage oi $\pi \rho \grave{\eta} \eta \mu \tilde{\omega} v$

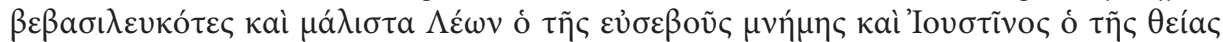
$\lambda \eta ́ \xi \varepsilon \omega \varsigma \grave{\eta} \mu \tilde{\omega} \nu \pi \alpha \tau \eta ̀ \rho . . . ~ a ̉ \pi \eta \gamma o ́ \rho \varepsilon v \sigma \alpha v,{ }^{19}$ wo sowohl Leon I. als auch Iustinos ordnungsgemäß angeführt werden, nur der Letztgenannte aber Iustinianos’ „Vater“ ist. Darüber hinaus werden hier beide ausdrücklich als Mitglieder der Gruppe der Vorgänger des Kaisers auf dem Thron bezeichnet, so dass „der Vater“ im Sinne des Vorgängers hier eigentlich eine Wiederholung des bereits Gesagten darstellen würde.

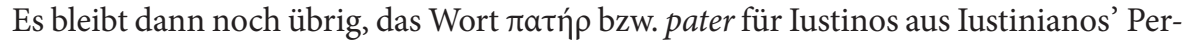
spektive als eine allgemeine Bezeichnung für einen (älteren und geehrten) Verwandten aufzufassen. Eine solche Verwischung der Grenzen zwischen zwei genealogischen Termini, Vater und Onkel, scheint zwar nicht besonders naheliegend zu sein, bleibt aber denkbar. Letzten Endes stand Iustinianos als Kaiser außer der Bestrebung um Verständlichkeit ja eigentlich nichts im Wege, seine Sprache bezüglich des Gebrauchs der Bezeichnung "Vater" auf diese Weise zu regulieren, aus welchem Grunde auch immer (etwa um der Selbstlegitimierung willen). Mindestens in diesem Sinne scheint Noethlichs' Relativierung der Belege für Iustinos als „der Vater“ des Iustinianos Bestand zu haben.

Als Kronzeugen von Iustinianos’ Adoption kann man jedoch den Beleg anführen, auf den Noethlichs nicht näher eingegangen ist. Es geht um Iustinianos' Erwähnung als „Sohn des universellen Kaisers" in einem Epigramm der Anthologia Graeca; die Inschrift mit ihm befand sich ursprünglich in einer konstantinopolitanischen Kirche und muss

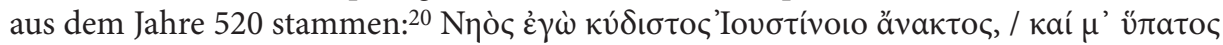

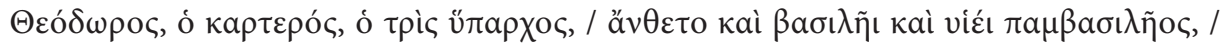

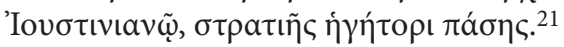

und auch von Av. Cameron (1975: 7-8) - vermerkt. Beide Quellen sind später als Corippus' Werk, doch geht es im Wesentlichen darum, dass eine solche Geschichte im Umlauf war.

17 Die Ausnahme: Novell. Iust. 28, 4, 2.

18 Auch wenn man Meiers (2003a: 144-145) These recht gäbe, dass Iustinianos’ Bewertung der Kaiser des 5. Jahrhunderts eindeutig abwertend war.

19 Novell. Iust. 109, praef. Die Übersetzung: „die vor uns Regierenden, besonders Leon seligen Andenkens sowie Iustinos, unser Vater frommen Andenkens, ... haben verboten." Vgl. auch die Juxtaposition des Zenon, des Anastasios und des „Vaters" Iustinos in Novell. Iust. 89, 7.

20 S. Al. Cameron (1976: 274 [zum Ort] und 279-280 [zur Datierung]).

21 Anth. Gr. I, 97. Die Übersetzung: „Ich bin die ruhmvolle Kirche des Herrschers Iustinos, und der Konsul Theodoros, der starke, der dreimalige Präfekt, hat mich sowohl dem Kaiser als auch dem Sohn des universellen Kaisers, Iustinianos, dem Führer des ganzen Heeres, geweiht.“" 
Der Text wird nicht aus der Perspektive des Kaisers (Iustinos) formuliert und berichtet objektiv, in der dritten Person; ${ }^{22}$ es ist hier somit nicht der Ort für die Verwendung des Lexems vióc als etwaiger Ausdruck von Iustinos' Zuneigung zu Iustinianos, ${ }^{23}$ wie groß man sich die Nähe des Stifters der Kirche und der Inschrift zu Iustinos auch vorstellen mag. Es ist auch kaum denkbar, dass hier irgendwelche unerhörten semantischen Experimente getrieben wurden (etwa die Benutzung des Lexems für einen jüngeren Verwandten). Der Text war doch der Kontrolle sowohl der Genannten als auch anderer Leser ausgesetzt, ${ }^{24}$ was nicht nur seine Verständlichkeit, sondern auch Sachlichkeit und Annehmbarkeit verlangte. Schließlich ist in diesem Fall, anders als bei den Texten von Iustinianos als Kaiser, auch chronologisch ausgeschlossen, dass wir mit einer nachträglichen Falsifizierung der Sachlage zu tun hätten.

Wie paradox das auch klingen mag, so bietet uns die spätantike epigrammatische Poesie also doch einen aus meiner Sicht hermeneutisch nicht anfechtbaren Beleg dafür, dass Iustinianos im Jahre 520 als Sohn des Kaisers gegolten hat. Und da er das leiblich nicht war, muss man an eine Form der Adoption seitens des Iustinos denken.

Damit haben wir auch einen terminus ante quem für die Adoption. Falls Iustinianos' Name als ein anderer Beleg für sie akzeptiert würde, könnte man sagen, dass der Akt bereits vor den September 518 datiert werden muss, ${ }^{25}$ ohne dass dadurch aber die Frage geklärt würde, ob Iustinos den Neffen vor oder aber nach seiner Thronbesteigung am 10. Juli jenes Jahres adoptiert hat. ${ }^{26}$

22 Darin sehe ich den grundsätzlichen Unterschied zu den Aussagen einiger anderen Quellen des 5.-6. Jahrhunderts, die als Sohn des Kaisers die Personen bezeichnen, die bestimmt nicht leibliche Söhne des Kaisers waren (bzw. umgekehrt den Kaiser als den Vater dieser oder jener Persönlichkeit anführen). Vgl. z.B. folgende Stelle des Briefes des Senats der Stadt Rom an den Kaiser Anastasios I. aus dem Jahre 516: animus domini nostri inuictissimi regis Theoderici filii uestri (Avell. 114, 1). Man könnte zwar die Möglichkeit erwägen, ob Theoderich nicht etwa von Anastasios adoptiert wurde, allerdings sicherlich nur durch die adoptio per arma - was in der Forschung nicht üblich ist -, oder die vermeintliche byzantinische „Familie der Könige“ als Erklärungsmodell heranziehen (dagegen neuerdings mit guten Gründen Brandes [2013: hier 269-270 mit Anm. 89 auch zu der zitierten Stelle]); vermutlich wurde hier aber Theoderich nicht als Sohn des Anastasios in irgendeinem adoptiven oder fiktiven Sinne präsentiert, sondern es wurde dem Kaiser von den Senatoren in konventioneller bzw. diplomatischer Sprache nur die Ergebenheit, Hochachtung o.ä. des Königs signalisiert. Das Wesentliche ist, dass der Kaiser dabei als der Adressat des Briefes (und somit auch der Bezeichnung von Theoderich als seinem Sohn) gedacht wurde. In der Novell. Theod. 2, tit. (Domino Valent(iniano) inclito victori ac triumphatori semper Augusto filio Theod(osius) p(er)p(etuus) A(ugustus) pater), um noch ein Beispiel mehr zu nennen, geht es um die Kommunikation, an der sogar die beiden Betreffenden, der "Vater" (der ältere und dienstältere Kaiser) Theodosios II. sowie der „Sohn“ (der jüngere Herrscher) Valentinianus III., teilnehmen. Der Auftraggeber des Epigramms Anth. Gr. I, 97 spricht dagegen nicht zum Kaiser über dessen „Sohn“, sondern zum beliebigen Leser über den Kaiser und dessen Sohn.

23 Eine Benutzung, die im allgemeinen Liddell, Scott, Jones (1996: 1847 [s.v. vióc 6]) erfassen („,as a general term of affection") und deren sich speziell für die Kaiser Al. Cameron (1976: 282) bewusst ist (,while it is true that emperors often addressed close colleagues and senior ministers as 'my son'").

24 Zutreffend bezog bereits Al. Cameron (1976: 282) die Tatsache ein, dass es um „a formal public inscription" ging.

25 Falls auf die Überschrift Avell. 147 (Exemplum epistolae Iustiniani) Verlass ist; zur Datierung s. den Apparat der Edition zur Stelle: Guenther (1895-1898: II, 592).

26 Die Frage der Datierung der Adoption hat Croke (2007: 20-21) gut bearbeitet; zu berichtigen sind allerdings die Angaben, dass Rosen (2001a: 765) die Adoption bereits ca. $505 \mathrm{n}$. Chr. datiere (so Croke 2007: 21 Anm. 39) - Rosen spricht an der Stelle (oder anderswo in seinem Artikel) überhaupt nicht von der Adoption - oder dass Vasiliev (1950: 93) sie mit Iustinos' Thronbesteigung verbinde (Croke 2007: 21 Anm. 40) - in Wirklichkeit scheint Vasiliev eher an die Zeit davor zur denken, explizit ist er aber jedenfalls nicht. Somit ist vielleicht der wichtigste Verteidiger der Datierung der Adoption 
Der Name des künftigen Kaisers, Iustinianos, ist aber an sich keine gesicherte Folge (und somit kein Beleg) einer Adoption. Die Argumentation von Croke mit der traditionellen Praxis („his name ... preserves the usual nomenclature of the adoption process, the addition of '-ianus' to the name of the adoptive father (as 'Octavius' became 'Octavianus' when adopted by Julius Caesar)"') 27 ist erstaunlicherweise fehlerhaft, da die mit -ianus gebildeten cognomina der Adoptierten ganz im Gegenteil an den ursprünglichen Namen (nomen gentilicium) erinnern sollten. ${ }^{28}$ Somit bleibt nur übrig, sich darauf zu berufen, dass Iustinianos' Name aus demjenigen seines Onkels abgeleitet wurde. ${ }^{29}$

Es steht zwar fest, dass wir mit der Derivationskette iustus (Sekundärwurzel iust-, thematischer Stamm iustu-) $\rightarrow$ iustinus (Suffix -inu-) $\rightarrow$ iustinianus (Suffix -ianu-) zu tun haben. Ob man aber bei der Namengebung im Fall des Iustinianos auf eine Person Bezug nahm, die Iustinus hieß, kann man nicht wissen. Falls Iustinianos wirklich erst sekundär diesen Namen angenommen hätte, wäre es natürlich ziemlich naheliegend, dass er dabei an den Onkel gedacht hat. Es ist aber ebenso möglich, dass „Iustinianos“ bereits sein Tauf- bzw. erster Name war, der von demjenigen des Onkels inspiriert und aus ihm abgeleitet worden war, ohne dass dies eine Adoption implizieren müsste (schon aus chronologischen Gründen, es sei denn, man möchte Iustinianos erst nach dem Tod des Vaters geboren und sofort vom Onkel adoptiert sehen). Und schließlich ist auch sehr wohl denkbar, dass dem Jungen der Name nach einem anderen Verwandten gegeben bzw. aus dem Namen dieses Verwandten abgeleitet wurde; so kann die Mutter Iustina oder der Großvater mütterlicherseits Iustinus oder aber auch Iustus geheißen haben. ${ }^{30}$

vor Iustinos' Kaisertum eben Al. Cameron (1976: 282): „the derivative name clearly implies ... that Justin legally adopted his nephew long before his own accession“. Das ist aber einfach unrichtig: der Name bzw. seine Absenz in den Quellen lässt zumindest Juli bis September 518 als durchaus mögliche Termini der Adoption bereits während Iustinos' Regierung übrig, ganz abgesehen von seiner Unverlässlichkeit als Indiz für den Akt. Ähnlich wie Cameron etwa noch Evans (1996: 62 mit 281 Anm. 217; anders aber 96 und 286 Anm. 2).

27 Croke (2007: 20).

28 Zur Problematik s. Shackleton Bailey (1976: 81-82) und Salway (1994: 127-128). Holmes (1912: 302) hat den Unterschied gesehen, da er aber bei der Argumentation bei der alten Praxis blieb, kam er zu folgendem Urteil: „the correct form would have been Justinus Sabbatianus, but the Byzantines were ignorant or varied old rules ad lib."

29 Die Logik der Argumentation für Iustinianos' Adoption mit Hilfe seines Namens hat, soweit ich sehe, so gut wie niemand hinreichend artikuliert. Andeutungsweise Vasiliev (1950: 93): „Justin legally adopted Sabbatius, who on this occasion assumed the derivative name of Justinian" oder Al. Cameron (1976: 282): „the derivative name clearly implies...".

30 Die spätantike Onomastik ist leider weitgehend unerforscht (vgl. Al. Cameron 1985: 164 und 171) und es ist von vornherein zu bezweifeln, dass die Quellen es erlauben würden, für die unteren Schichten der Gesellschaft im Balkan des 5. Jahrhunderts klare onomastische Regeln festzustellen. Immerhin ist es im allgemeinen bereits für den Prinzipat und den romanisierten Teil des Reiches keineswegs möglich, davon auszugehen, dass die cognomina auf -ianus unbedingt von einer Adoption zeugen; ,in most cases it is better to assume that these cognomina were derived from names of relatives or friends (etc.)“; s. Salomies (1992: 61 [das Zitat in Anm. 2]). Zum Wesen der spätantiken Namengebung, auch der niederen Schichten, vgl. jedoch Salway (1994: zusammenfassend 144-145): das System beruht nominell noch auf nomen (gentilicium) und cognomen, praktisch ist für die meisten Bewohner des Reiches aber nur das Letztgenannte wichtig. Wie Iustinianos' Mutter hieß, ist unbekannt; s. z.B. Vasiliev (1950: 59). Zur Möglichkeit, dass der fragliche Verwandte Iustus hieß (und Iustinianos' Name ohne Bezug auf das Mittelglied der Derivationskette gebildet wurde), vgl. etwa die Tatsache, dass zwei Brüder im 4. Jahrhundert, die späteren Kaiser, die Namen Valentinianus und Valens bekommen haben; der Vater, übrigens ein aus Pannonien stammender Mann unvornehmen Ursprungs (PLRE I: 400-401 [s.v. Gratianus 1]), hat also, soweit man sehen kann (vgl. PLRE I: 1130 [Stemma 4]), den Mittelglied der Derivationskette, Valentinus, bei der Namengebung übergangen. Zu beachten ist auch, dass in der 
Diese zwei Interpretationen von Iustinianos' Namen widersprechen dem tradierten und bisher wahrscheinlich nie bezweifelten Wissen, dass Iustinianos ursprünglich anders geheißen habe, nämlich Petrus Sabbatius, ${ }^{31}$ was sich auf die Inschrift von Iustinianos' Konsulardiptychen aus dem Jahre 521 mit dem folgenden Vollnamen stützt: Fl(avius) Petr(us) Sabbat(ius) Iustinian(us).32 Seine übliche Deutung, falls man überhaupt von einer solchen sprechen kann, scheint mir aber unrichtig zu sein.

Die Quellen suggerieren, dass Iustinos aus recht armen und einfachen Verhältnissen stammte. Obwohl sie alle nicht unproblematisch sind, ${ }^{33}$ ist doch kaum daran zu denken, dass Iustinos und per analogiam auch sein $\mathrm{Neffe}^{34}$ in der ursprünglichen sozialen Umgebung Anlass gehabt hätten, sich den Namen so zu gestalten bzw. einen solchen nach der Geburt zu erhalten, wie ihn die Konsulardiptychen für den Letzteren bezeugen. Das setzt auch die übliche Sichtweise voraus (mindestens „Iustinianus“ müsste die spätere Zugabe sein), und im allgemeinen bestätigt das auch das erste Glied der Namensfolge, „Flavius", ein seit dem 4. Jahrhundert (zumindest im östlichen Teil des Reiches) effektiv zum Ausdruck des Status des Trägers transformiertes nomen gentilicium, das sich Iustinianos sicherlich im Jahre 518 (noch vor Thronbesteigung des Onkels) als Mitglied der candidati $i^{35}$ beigelegt haben dürfte, kaum aber bereits in seiner Jugend. ${ }^{36}$

Üblicherweise sieht man den für das Jahr 521 verzeichneten Vollnamen des künftigen Kaisers als etwas infolge der Adoption Gewordenes und, indem man dann von Fl(avius) absieht und Iustinian(us) als sekundär betrachtet, glaubt man, in Petr(us) Sabbat(ius) die

Familie des Iustinos und des Iustinianos der Name Iustus wirklich belegt ist: s. PLRE III: 758-759 (s.v. Iustus 2 und Iustus 3) und zur Anschaulichkeit vgl. auch PLRE II: 1315 (Stemma 10).

31 S. z.B. Vasiliev (1950: 61), Evans (1996: 96), Croke (2007: 20), Leppin (2011: 29). Holmes (1912: 301) spricht ohne irgendwelcher Begründung nur von Sabbatius, Petrus ignorierend; Vasilievs (1950: 93) einmaliges „Sabbatius“ ist dagegen wahrscheinlich nur stilistisch bedingt.

32 CIL V 8120, 3 und XIII 10032, 7 (FL. PETR. SABBAT. IVstinian. usw.). Erstgennante Nummer wird in der Literatur des öfteren falsch als CIL V 8210, 3 zitiert, zum ersten Mal wahrscheinlich von Bury (1923: 19 Anm. 6), dessen Fehler schon Vasiliev (1950: 62 Anm. 40) bemerkt hat.

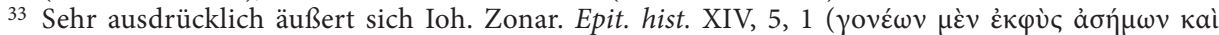

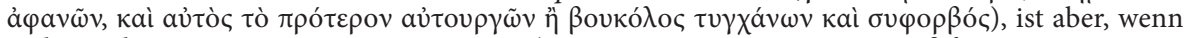

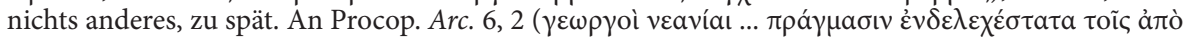

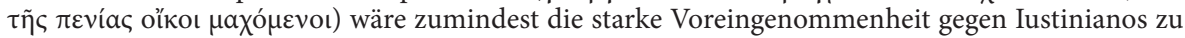
bemängeln. Und den Bericht bei Ps.-Zachar. Schol. Hist. eccl. VIII, 1 könnte man, wie ich glaube, auch als ein Produkt der Selbststilisierung und Propaganda des Iustinos verdächtigen.

34 Kaum denkbar ist, dass Iustinianos' Vater ein zumindest in lokalen Verhältnissen prominenter Mann war. Da Iustinos noch unter Leon I. (457-474) nach Konstantinopel kam (Procop. Arc. 6, 2) und Iustinianos möglicherweise um 482 geboren wurde (so zumindest nach Georg. Cedren. Compend. hist. I, 642 [de Boor] und Ioh. Zonar. Epit. hist. XIV, 5, 40), konnte vielleicht die Familie des Neffen aus Iustinos' Karriere schon im Jahre 482 einigen Nutzen ziehen, einen wesentlichen gesellschaftlichen Aufstieg bedeutete das für sie aber damals sicherlich noch nicht; Procop. Arc. 6, 4 weiß jedenfalls mehr von Iustinos' Karriere erst unter Anastasios I. (491-518) zu berichten.

35 Iustinianos als candidatus bei der Wahl des Iustinos belegt Const. Porph. De cer. I, 93 (428 Reiske) und chronologisch nicht so ganz eindeutig auch Vict. Tonn. Chron. ad a. 518, 2.

36 Zur Problematik des Flavius-Namens s. Keenan (1973: besonders 56-63 [Übersicht der Kategorien der Träger im Ägypten des 5.-6. Jahrhunderts] und 63 [Zusammenfassung: ,anyone, who held an imperial dignitas, honor, or administratio, or who had been approved for service in an imperial militia ... or who held certain civic magistracies, was entitled to the name Flavius"]) und Salway (1994: 137-138 und 140-141). Zum Unterschied zwischen den westlichen Aristokraten und den Eliten des östlichen Teils des Reiches bei der (Nicht)handhabung des Flaviusnamens vgl. auch Al. Cameron (1988: 30-31 und 33). 
ursprüngliche Bezeichnung vor sich zu haben. ${ }^{37}$ Die notwendige Voraussetzung, dass Söhne armer und niedrig stehender Eltern im Balkan des 5. Jahrhunderts zwei Namen hatten, wäre aber kaum mit irgendeiner passenden Parallele zu untermauern. Sehr wichtig ist, dass Iustinianos' Vater (im Lateinischen) Sabbatius hieß. ${ }^{38}$ Will und kann man behaupten, dass Petrus Sabbatius nach der Geburt auch den Namen des Vaters bekam, und zwar als das zweite und zugleich letzte Glied des Vollnamens?

Wenn man die Tatsache berücksichtigt, dass die Konsulardiptychen die einzigen Belege für den vollständigen Namen von Iustinianos bieten, kann man dazu neigen, ihn als die Schöpfung erst des Moments aufzufassen, in dem sich Iustinos' Neffe das Konsulat gesichert hat. Wie dem auch sein mag (und auch berücksichtigt, dass möglicherweise Iustinianos gelegentlich schon früher, besonders in den Jahren 518-520 nach Iustinos' Thronbesteigung, die Gelegenheit und Mittel dazu hätte haben können, sich mit diesem oder zumindest ähnlichem Vollnamen zu präsentieren), so scheint eines aber sicher zu sein: es geht um den Namen eines Mannes, der sogar bereits zu den höchsten Kreisen Konstantinopels und somit des (östlichen Teils des) Reiches gehört, wie sich nicht etwa an „Flavius“, 39 sondern an der Benutzung dieses Namens in den Konsulardiptychen und

37 So wahrscheinlich die Überlegungen von Bury (1923: 19 einschließlich der Anm. 6), Vasiliev (1950: 61) oder Leppin (2011: 29 mit 357 Anm. 2), die allerdings immer nur implizit bleiben.

38 Es gibt dazu zwei Quellen: Procop. Arc. 12, 18 und Theoph. Chron. ad a. 6024 (183 de Boor). Dass jener angebliches Gerede referiert, Iustinianos sei eben nicht Sohn des Sabbatios, sondern eines Dämons, ist sicherlich kein Problem. Ernsthafter ist, dass die einschlägige Erwähnung bei Theophanes im Rahmen des notorischen und mit anderen großen Problemen (hinsichtlich der Überlieferung und Quellen der Chronik von Theophanes und der Osterchronik sowie in Bezug auf den rätselhaften „Megas Chronographos“ oder überhaupt den Nika-Aufstand) verbundenen Zirkusdialogs erscheint, der auch unter der Bezeichnung Akta dia Kalopodion bekannt ist. Es ist nicht möglich, diese Fragen hier zu erörtern, so dass ich mich mit der Feststellung begnüge, dass die Datierung des Textes im Rahmen von Iustinianos' Regierung umstritten ist, und mit dem Verweis auf die neueste mir bekannte Behandlung der Problematik von Meier (2003b: 278-285), die einen ausführlichen und die frühere Literatur miteinbeziehenden Überblick bietet. Beide Quellen werden z.B. von Vasiliev (1950: 60) oder PLRE II: 966 (s.v. Sabbatius) ordnungsgemäß angeführt, die zunächst überraschende Tatsache aber, dass Prokopios den Namen des Vaters in der

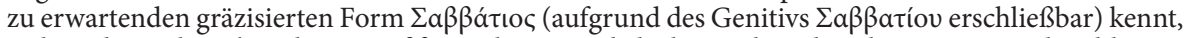
während man bei Theophanes $\Sigma \alpha \beta \beta a ́$ đๆ ఢ liest, wurde bisher nicht näher thematisiert - obwohl mindestens Leppin (2011: 357 Anm. 4) den Leser auf den Unterschied aufmerksam macht. Dabei könnte die (in der Literatur stillschweigend kaum beachtete) Form des Theophanes die größere Autorität beanspruchen, da der Text des Zirkusdialogs in der Chronographie, wie mir scheint, wahrscheinlich letzten Endes auf der Aufzeichnung in einem offiziellen oder offiziösen Dokument beruhen dürfte. In Wirklichkeit ist allerdings die Form des Namens bei Theophanes nur eine „vulgärgriechische“ Modi-

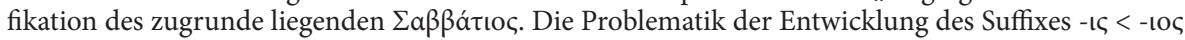
(bereits seit dem 3. Jahrhudert v. Chr. und nebst dem neutralen Gegenstück -ıov > -ıv [und zuletzt $>-1]$ ) ist bisher leider nicht befriedigend erklärt; vgl. dazu immerhin die forschungsgeschichtlich informative und die byzantinischen Chroniken (einschließlich jener des Theophanes) berücksichtigende Behandlung von Psaltes (1913: 43-48) sowie Gignac (1981: 25-29). Die somit ursprünglichere Lesung $\Sigma \alpha \beta \beta a ́ \tau \iota c ̧$ ist in einem Teil der handschriftlichen Überlieferung von Theophanes' Chronographie erhalten (vgl. den Apparat der Edition von de Boor zur Stelle [S. 183]) und den Text hat in dieser Hinsicht Maas (1912: Nr. I, Z. 51 [S. 398]) besser ediert. Die jüngere Form des Suffixes war im Namen des Vaters von Iustinianos wahrscheinlich bereits bei der Zirkusdeklamation benutzt worden und geht somit nicht erst auf den Autor der Chronographie oder einen Kopisten dieses Werkes zurück - und

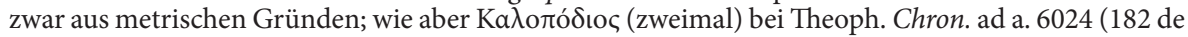
Boor) bzw. in Maas (1912: Nr. I, Z. 15-16 [S. 396]) zeigt, war sie in der Sprachvarietät der damaligen "Grünen" oder mindestens ihres mandator vermutlich noch nicht verallgemeinert.

39 Mit den Worten von Al. Cameron (1988: 33): „as common as dirt in the lower reaches of the new municipal elites." 
vor allem an seinem mehrgliedrigen (polyonymen) Charakter zeigt. Man sollte ihn deshalb unter Vergleich mit den Namen der Persönlichkeiten aus diesen Kreisen analysieren.

Die Polyonymie der Eliten des östlichen Reichsteils 5.-6. Jahrhunderts ist zwar leider ebenfalls nicht detailliert erforscht, das bisher Geleistete (vor allem in den letzten zwei bis drei Jahrzehnten $)^{40}$ scheint mir aber für die Bedürfnisse dieses Beitrags ausreichend zu sein. Im Allgemeinen kann man sagen, dass die einzelnen Glieder des Vollnamens, vom gentilicium bzw. Statusnamen Flavius und dem eigentlichen (diakritischen) Namen des Trägers abgesehen, entweder Namen der Ahnen bzw. Verwandten oder der weltlichen Patrone oder sog. christliche bzw. devotionale Namen (christologische, marianische, der Heiligen usw.) waren. ${ }^{41}$

Der östliche Konsul des Jahres 521 hatte an der vorletzten Stelle seines Vollnamens das cognomen des (leiblichen) Vaters. ${ }^{42}$ Das war in den polyonymen Reihen der östlichen Eliten üblich. ${ }^{43}$ Immerhin ist dann auch Folgendes naheliegend: „Les polyonymies des dignitaires ... sont plutôt des cumuls d'anthroponymes et non des formules onomastiques dans le plein sens du terme. Loin de faciliter l'identification d'un individu, ces cumuls ont plutôt pour objet de faire étalage de ses liens de famille et de ses appuis politiques." 44 Wenn aber so viel von den Namen der Männer aus der Oberschicht gilt, ist es höchst unwahrscheinlich, dass der wenig vornehme Iustinianos nach der Geburt - und überhaupt bevor er sich den durch die Diptychen überlieferten oder einen ähnlichen Vollnamen beilegte - wirklich auch den Namen des Vaters getragen hatte und mit ihm sogar im Alltag angeredet worden war.

Die übliche Hypothese könnte man aber schwerlich auch in einer eventuellen modifizierten Form retten, nämlich dass Iustinianos vor der Adoption bloß Petrus bzw. Petros hieß. Zwar kann man Sicherheit nicht erlangen, da uns andere zur Vergleichung geeignete Fälle von polyonymen Namen bekanntermaßen adoptierter Personen aus dem 5.-6. Jahrhundert fehlen, doch ist es unwahrscheinlich, dass zwei persönliche Namen, der ursprüngliche und derjenige nach der Adoption angenommene, in der polyonymen Reihe durch die cognomina der Verwandten getrennt worden wären. ${ }^{45}$

40 S. Feissel, Kaygusuz (1985: 230-233), Salway (1994: 141-143), Gascou (2002: 431-432), Laniado (2002: 136; 2004: 328-334; 2012), Feissel (2004: 299 und 313-314).

$41 \mathrm{Zu}$ den Ahnen und Verwandten besonders Salway (1994: 141-142) und Laniado (2012: 34-39); zu den Patronen zuerst Gascou (2002: 431 Anm. 8) und danach Laniado (2004: 329-333 und 2012: 39-42); zu den christlichen Namen vor allem Salway (1994: 142-143) und Laniado (2012: 42-49), vgl. aber anhand eines epigraphischen Neufundes mit dem Vollnamen des verrufenen Prätoriumspräfekten Ioannes von Kappadokien auch Feissel (2004: 299).

42 Es sei hier erwähnt, dass dies im richtigen Kontext der polyonymen Namen zumindest Feissel, Kaygusuz (1985: 233 Anm. 18) gesehen und erwähnt haben.

43 In diesem Sinne Feissel, Kaygusuz (1985: 232 einschließlich Anm. 17), Salway (1994: 141) und Feissel (2004: 299 einschließlich Anm. 247). Laniado (2004: 329) gibt das ebenfalls zu, obwohl er, sicherlich berechtigt, mit Verweis auf Ausnahmen zur Vorsicht bei der Interpretation der Namen von genealogisch sonst nicht näher bekannten Persönlichkeiten mahnt (Laniado 2004: 342).

44 Laniado (2004: 334).

45 Der Fall von Tiberios II., der im Jahre 574 vom Kaiser Iustinos II. adoptiert, als Konstantinos benannt oder vielleicht eher umbennant und zum Caesar ausgerufen wurde (s., auch zu den Quellen, PLRE III: 1323 und 1325 [s.v. Tiberius 1]), um dann zumindest offiziell den polyonymen Namen Fl. Tiberios Konstantinos zu tragen (s. vor allem den Titel der von Kaplan [1981: 238-241, hier Z. 3-4] neu edierten Novelle, die Tiberios bereits als Augustus nach 578 erließ), bietet auf den ersten Blick eine erwünschte Analogie. Dieser Schein trügt jedoch. Auch abgesehen davon, dass diese Namensänderung oder -ergänzung (s. auch die das Bild noch komplizierende Überlegung von Whitby 1994: 90-91) in den 
Wie kann man Petr(us) in Iustinianos' Vollnamen aber anders interpretieren? An sich steht dem nichts im Wege, hier einen weiteren von einem Verwandten übernommenen Namen zu sehen, am ehesten den von Iustinianos' Großvater. ${ }^{46}$ Weitaus wahrscheinlicher scheint mir aber zu sein, dass wir einen devotionalen Namen vor uns haben. Iustinianos hat in Konstantinopel eine Petrus und Paulus geweihte Kirche bauen lassen und im Jahre 519 den Papst Hormisdas um die Reliquien dieser Apostel und des heiligen Laurentius für sie gebeten, ${ }^{47}$ so dass an seinem Willen zu dieser Zeit, die Frömmigkeit gegenüber Petrus zur Schau zu stellen, kaum Zweifel bestehen können. Natürlich wird unbekannt bleiben, wie aufrichtig und tief jene mit dem Kirchenbau (sowie, wie man jetzt sagen kann, dem neuen Petrusnamen) bezeugte Frömmigkeit war. Es wäre möglich, sie lediglich (oder auch) als eine Strategie aufzufassen, als entschiedener Anhänger der Inhaber des Petrusstuhles und somit als Verteidiger des Konzils von Chalkedon zu posieren. In der Zeit vor und nach der Beilegung des akakianischen Schismas im Jahre 519, als Iustinianos' wichtigster Konkurrent (eventuell auch bei der Sicherung der Throns nach Iustinos' Tod), Vitalianus, ${ }^{48}$ unter den Chalkedonianern immer noch als der vermutlich populärste Parteigänger galt, ${ }^{49}$ konnte das für Iustinianos’ Reputation sicherlich vorteilhaft sein. Im Lichte dieser Überlegungen dürfte man sehr wohl den Augenblick der Annahme des Namens Petros im Rahmen der neugebildeten, uns erst aus den Konsulardiptychen bekannten polyonymen Reihe von Iustinianos als Neffen (und möglicherweise bereits als Sohn) des Kaisers Iustinos schon auf die Zeit ganz am Anfang der Regierung

Quellen nicht ausdrücklich mit der Adoption verknüpft, sondern eher durch Tiberios' Herrschaftsantritt motiviert wird (Ioh. Ephes. Hist. eccl. [pars tertia] III, 5 [S. 95 der Übersetzung von Brooks]: „Tum eum apparatu et vestitu et habitu regni vestivit, dicens ei: « Dehinc nomen tuum vocabitur Constantinus, pro eo quod in te regnum Constantini magni renovatum est " ") - ohnehin ist der neue Name nicht von jenem des Adoptivvaters abgeleitet -, enthält die polyonyme Reihe dieses Kaisers (deren Existenz an sich schon einen Unterschied von Iustinianos und den früheren Kaisern darstellt; vgl. weiter unten S. 214-215) nur den ursprünglichen und den neuen persönlichen Namen, darüber hinaus aber kein weiteres cognomen, aus dessen Position man dann eventuell Rückschlüsse über den Aufbau von Iustinianos' Vollnamen ziehen könnte.

46 Eher väterlicherseits, und zwar nicht so sehr deshalb, weil man als den Namen des Großvaters mütterlicherseits einen Iustus oder Iustinus vermuten kann (s. oben S. 206), sondern eher, weil man hier eher die Nennung des Vaters des in der Namenreihe folgenden Sabbatius erwarten würde als die eines Ahnen aus der anderen Geschlechtslinie.

47 Der Bau: Procop. Aed. I, 4, 1-2; die Bitte: Avell. 187, 5-6 (zur Datierung vgl. den Apparat der Edition: Guenther 1895-1898: II, 644) und 218 (zur Datierung vgl. den Apparat der Edition: Guenther 1895-1898: II, 675).

48 S. PLRE II: 1171-1176 (s.v. Vitalianus 2) und vgl. die Bewertung von Croke (2007: 33 [„By now Vitalian was not only the most powerful man at court after the emperor, but he had immediately succeeded Justin as consul for 520.“], 33-34 und 56), Leppin (2011: 74 [„,der mächtigste Konkurrent schien unangefochten: Vitalian“]) sowie Greatrex (2007: 105 [„Justinian ... was the principal rival of the erstwhile rebel“]).

49 Zumindest die Synoden im Osten des Reiches im Jahre 518 haben uns, wie es scheint, dafür Belege

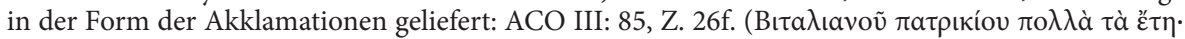

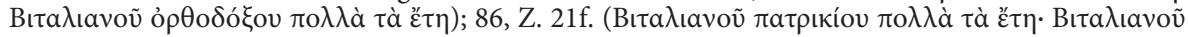

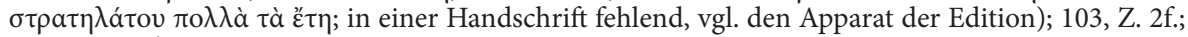

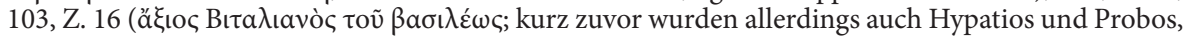
zwei der Neffen des Kaisers Anastasios, akklamiert). Vgl. Croke (2007: 27 [„By September 518 it was clear to the bishops, clerics and monks of the eastern provinces that Vitalian was the next most important figure to Justin and Euphemia, because of his championing of orthodoxy"]). 
des Letztgenannten datieren: im Juli 518 hatten nämlich die Ereignisse begonnen, die zum Ende des Schismas führten..$^{50}$

Gegen die Interpretation des fraglichen $\operatorname{Petr}(u s)$ in Iustinianos' Konsulardyptichen als devotionaler Name könnte man einwenden, dass diese Kategorie der cognomina der polyonymen Reihen erst später bezeugt ist, und zwar zuerst im Jahre $533 .{ }^{51}$ Das ist aber kein schwerwiegendes Hindernis. Im Gegenteil würde Iustinianos' Fall auch unter diesen Umständen sogar eine Antwort auf die Frage erlauben, wie die Mode der christlichen cognomina entstehen und sich durchsetzen konnte. Dass sich Iustinianos einen devotionalen Namen für seine polyonyme Reihe zwischen 518 und 520 als überhaupt erstes Mitglied der konstantinopolitanischen Elite gewählt hat und zunächst damit vereinzelt geblieben ist, wäre im Lichte seines (späteren) Rufs eines gewaltigen Neuerers ${ }^{52}$ denkbar und mit Rücksicht auf die oben vermutete spezifische, situationsgebundene Motivation auch verständlich. Als er aber immer prominenter wurde und spätestens, nachdem er Kaiser geworden war (obwohl er sich vielleicht gerade damals den eigentlichen Namen auf Iustinianos eingeschränkt hatte), ${ }^{53}$ musste sein früherer Schritt für einige durchaus nachahmenswert erscheinen. Einer der zwei ersten belegten Träger von devotionalen Namen ist der praefectus praetorio per Orientem Ioannes von Kappadokien, ein Mann, dessen ziemlich niedrige Herkunft zwar nicht ausdrücklich bezeugt ist, sich aber vermuten lässt. ${ }^{54}$ Die Annahme liegt also nahe, dass er sich, als er Prätoriumspräfekt geworden war und einen entsprechend glänzenden (d.h. polyonymen) Namen für die Akten (subjektiv) brauchte, jedoch keine imponierende Ahnenreihe für dessen Bildung besaß, auf den früheren Namen des Kaisers besann, der ebenfalls wenig vornehmer Herkunft war. ${ }^{55} \mathrm{Er}$ seinerseits konnte damit andere, zunächst vor allem seine Untergebenen, inspirieren; und einige der vornehmeren und prominenteren Glieder

50 S. dazu Stein (1949: 223), Croke (2007: 26-27) oder Leppin (2011: 54-58).

51 Und zwar im epigraphisch überlieferten und von Feissel (2004: 262-266) edierten Dossier drei Dokumente aus Didyma, von denen die ersten zwei eine Datierung enthalten (1. und 2. April 533) und das letzte von Feissel (2004: 270) ebenso auf das Frühjahr 533 datiert wird. Ein Name wird in Z. 42 (zweiter Dokument), andere in Z. 57 (dritter Akt) genannt; s. weiter Anm. 56. Die erste Person, der Prätoriumspräfekt Ioannes von Kappadokien, erscheint in Z. 46 auch unter einer kürzeren, aber immer noch grundsätzlich polyonymen Version des Namens, Fl(avius) Marianus Ioannes.

52 Zusammenfassend Noethlichs (2000: 128-129 und 131-132).

53 In den offiziellen Urkunden des Kaisers findet man im Rahmen der vollen Titulatur den Namen vor dem 6. Juni 533 bloß als Iustinianus (Cod. Iust., De cod. comp. [Const. Haec], tit. [der allzu einfache Titel dieser Konstitution vom 13. Februar 528 ist jedoch kaum authentisch]; Cod. Iust., De cod. confirm. [Const. Summa], tit.; Cod. Iust. I, 1, 8, 7 = Avell. 84, 7 = Avell. 91, 8), seit dem 21. November 533 dann als Flavius Iustinianus bzw. als dessen griechisches Äquivalent (Inst. Iust., De inst. promulg. [Const. Imperatoriam], tit. usw.). Zur Problematik (der Grund der Namensänderung) s. auch weiter unten S. 213-215.

54 Seine Herkunft berührt noch am ehesten Ioh. Lyd. Mag. III, 57, 2, beschränkt sich aber auf die geographischen Angaben. Zumindest seine Bildung bemängelt Procop. Pers. I, 24, 12. An unvornehme Herkunft des Mannes denken eben im Zusammenhang (auch) mit seinem polyonymen Namen Feissel (2004: 299) und Leppin (2011: 122-124).

55 Iustinianos kann übrigens seinerzeit zusätzlich zur Schaustellung der Frömmigkeit gegenüber Petrus und der Treue zu den römischen Patriarchen und zum Chalkedon ähnlich motiviert gewesen sein, und Flavius Sabbatius Iustinianus wäre ja vermutlich nicht hinreichend polyonym gewesen. Ioannes wurde Präfekt zum ersten Mal zwischen Februar und April 531 (PLRE III: 628 [s.v. Ioannes 11]). Immerhin war er vorher scriniarius in der Kanzlei des magister militum gewesen, möglicherweise gerade unter Iustinianos, als dieser das Amt des magister militum praesentalis innehatte (520-527), wie in PLRE III: 627 (s.v. Ioannes 11) vermutet wird. Obwohl also in den Jahren 527 (wenn nicht sogar ein bisschen früher) bis 531 devotionale Namen vermutlich noch kein Trend waren, ja möglicherwei- 
der Gesellschaft ließen sich wahrscheinlich nicht an religiösem Eifer von einem Ioannes von Kappadokien überbieten und fügten sich daher devotionale cognomina zu den von Ahnen oder weltlichen Patronen abgeleiteten hinzu. ${ }^{56}$ Diese Rekonstruktion bleibt

se es keinen Träger solcher Namen gab, hätte der neue Präfekt Ioannes gute Voraussetzungen dazu
gehabt, sich des ehemaligen Petrusnamens des Iustinianos bewusst zu sein.
Der Name des Präfekten war Fl(avios) Mariano $<$ s $>$ Micael(ios) Gabriel(ios) Arcangel(ios) Ioannes;
der in der Inschrift aus dem Jahre 533 zugleich bezeugte Statthalter von Karien Fl(avius) Mar(ianus)
M<ic $>$ hael(ius) Gabriel(ius) Ioannes $<$ E $>$ utropi<u $>$ (s. Anm. 51) hatte mit ihm die Reihe der drei
sicherlich devotionalen cognomina Marianos Michaelios Gabrielios gemeinsam, die sich dann auch
in den Namen von sechs Persönlichkeiten aus der Zeit nach der Hälfte des 6. Jahrhunderts vorfin-
den (zum Material s. Feissel, Kaygusuz 1985: 225 [Z. 1-2 der edierten Inschrift] und 231-232 oder
Laniado 2012: 46-47), die Ämter, sei es in der zentralen, sei es in der regionalen Verwaltung, inne-
hatten. Zumindest im Fall des Eutropius lässt sich aus chronologischen Gründen sehr wohl auf die
Übernahme der Namendreiheit von Ioannes dem Kappadoker denken, umso mehr, als sein vorletztes
cognomen Ioannes auf den Präfekten als Förderer o.ä. verweisen kann, was auch Feissel (2004: 314 )
erwägt. Aber auch einige der übrigen uns bekannten Beamten können sich diese drei Namen beigelegt
haben, als der Kappadoker noch im Amt war. Vgl. zum Ursprung und zur Ausbreitung der devotionalen cognomina auch die Beobachtungen von Laniado (2012: 45 und 48).

Iustinos, ein Sohn von Iustinianos' Cousin Germanos, trägt in seinen Konsulardiptychen (für das Jahr 540) den Namen FL. MAR PETR THEOdOR VALENT. RUST. BORAID. GERM. IUST. (CIL XIII 10032, 9). Feissel, Kaygusuz (1985: 232) haben vorgeschlagen, das erste cognomen als Marianus zu identifizieren. PLRE III: 750 (s. v. Iustinus 4) hält implizit auch ein profanes cognomen für weiterhin denkbar, der Verweis auf die (diakritischen) Namen Marcellus und Marcianus in der kaiserlichen Familie ist aber nicht überzeugend, beide einschlägige Persönlichkeiten (s. PLRE III: 816-817 [s. v. Marcellus 5] und 821-823 [s. v. Marcianus 7]) gehören derselben Generation an wie Iustinos selbst; es ist also unwahrscheinlich, dass Iustinos' cognomen auf einen von ihnen Bezug genommen hat; und auch wenn man einen früheren Träger des fraglichen Namens in der Familie vermuten würde, wäre es schwerlich jemand aus dem Zweig, dem Iustinos angehört hat (vgl. PLRE II: 1315 [Stemma 10]), so dass der Letztgenannte keinen besonderen Grund gehabt hätte, jenen Verwandten in seine polyonyme Reihe aufzunehmen.

Iustinos hat also, wie es scheint, mindestens ein devotionales cognomen. Als Mitglied der Dynastie brauchte er sich nicht Ioannes von Kappadokien zum Vorbild zu nehmen (und selbst wenn er oder vielmehr der Schöpfer seines vollen Namens, etwa sein Vater, sich bei Ioannes und nicht einfach von der sich bereits verbreitenden Mode hätte inspirieren lassen, hätte er das eher nicht zugegeben). Dementsprechend findet man in dieser Reihe Michaelios oder Gabrielios nicht.

War auch Iustinos' zweites cognomen Petr(us) devotional? Wenn nicht, würde es vermutlich auf einen Verwandten namens Petros Bezug nehmen, also kaum einen anderen als Iustinianos, und somit dafür sprechen, dass der Kaiser doch ursprünglich Petrus geheißen hat. Diese Möglichkeit scheint mir aber unwahrscheinlich, schon deshalb, dass nur ein einziges devotionales cognomen in einer längeren polyonymen Reihe eine Rarität gewesen wäre (doch ist Fl. Menas Iustinianos Demosthenes Ioannes Thomas mit „Menas“ wahrscheinlich ein Beispiel; s. Laniado [2004: 331-332; 2012: 42] und zu der Persönlichkeit PLRE III: 1316-1317 [s.v. Thomas 10]). Außerdem wäre es bestimmt ziemlich deplatziert gewesen, den kaiserlichen Verwandten mit dem Namen ehren zu wollen, den dieser seit Jahren nicht mehr getragen hatte. Es geht am ehesten um die Schaustellung seiner Frömmigkeit gegenüber dem heiligen Petrus (die zugegebenermaßen vom ehemaligen Präzedenzfall des Iustinianos inspiriert worden sein kann).

Schließlich folgen Petr(us) in Iustinos' Vollnamen weitere cognomina Theodor(us), Valent(inus) und Rust(icus) oder Rust(icius), die ich ebenfalls eher als devotional interpretieren möchte. Feissel, Kaygusuz (1985: 233 Anm. 18) vermuten zwar, es geht um „trois noms probablement entrés par alliance dans la famille impériale“, aber es fällt nicht leicht, in Iustinos' Stammbaum den Platz für etwa drei solche angeheiratete Verwandte zu finden, die, obwohl sonst unbezeugt, dazu noch so bedeutend gewesen sein müssten, dass sie der Schöpfer der polyonymen Reihe des Konsuls des Jahres 540 der Eingliederung für wert erachtet hätte. Meyer (1881: 9) hat seinerzeit geglaubt, dass sich Theodor(us) auf die Kaiserin Theodora, die Gemahlin des Iustinianos, bezieht. Das klingt zwar auf den ersten Blick einleuchtend und lässt sich mit Sicherheit in diesem konkreten Fall - es geht ja um einen Angehörigen der kaiserlichen Familie - nicht ganz ausschließen, doch ist zu beachten, dass dies für Laniado 
hypothetisch, und natürlich muss die neue Namensgebungspraxis nicht oder nicht ausschließlich vom Prätoriumspräfekten ihren Anfang genommen haben; dass sie aber vom früheren außergewöhnlichen Schritt des Iustinianos inspiriert wurde, nachdem dieser als Kaiser sozusagen nachahmenswert geworden war, scheint mir als Erklärung des zeitlichen Abstands zwischen dem devotionalen Namen des Iustinianos und solchen aus dem Jahre 533 hinreichend.

Die bisherige Diskussion kann man folgendermaßen zusammenfassen: Während die Bezeichnung des Kaisers Iustinos I. als „Vater“ seitens seines Nachfolgers für die Frage nach Iustinianos' Adoption nichts Verbindliches bietet, darf man aufgrund eines Epigrammes der Anthologia Graeca diesen Akt doch postulieren und ihn vor 520 datieren. Der Name Iustinianos hat aber entgegen der herkömmlichen Meinung mit jener Adoption nichts zu tun, da ihn der Kaiser bereits seit der Geburt getragen hat. Umgekehrt waren Petrus und Sabbatius nur sekundäre cognomina in der polyonymen Namenreihe, die sich Iustinianos erst als Mitglied der konstantinopolitanischen Elite beigelegt hat. Der Petrusname ist als implizite Deklaration der Treue zum Papst und Konzil von Chalkedon zu bewerten, was man (auch) als ein Instrument zur Sicherung der bedeutenden Stellung und der Thronfolge ansehen kann.

Es wird nützlich sein, in diesem Zusammenhang anhangsweise noch auf die Problematik von Iustinianos' Verzicht auf die Namen Sabbatius und Petrus einzugehen, den man bisher dadurch erklärt hat, dass Iustinianos nicht besonders stolz auf seinen Vater war ${ }^{57}$ oder dass ihn die heidnischen Konnotationen des Namens Sabbatius gestört hatten. ${ }^{58}$ Beides hilft jedoch wenig: der Petrusname war weder heidnisch gefärbt noch hatte er mit Iustinianos' Vater etwas zu tun, trotzdem hat der Kaiser auf jenen ebenso verzichtet. Die erstgenannte Hypothese ist schon dadurch erheblich geschwächt, dass man einräumen müsste, dass Iustinianos noch als Konsul des Jahres 521 mit diesem Namen nicht so große Probleme gehabt zu haben scheint. Und auch die heidnischen Konnotationen von "Sabbatius“ scheinen mir sehr unsicher zu sein: der Name kann in der bezeugten Form (vor allem in den Konsulardiptychen) kaum zum heidnischen Gott Sabazios eine Beziehung haben, in dessen Namen die Grapheme $<-b b->$ und $<-t->$ (bzw. die dadurch wiedergegebenen Laute) sehr ungewöhnlich wären, 59 auch wenn eine solche Orthographie (bzw. Lautung) des Namens im Balkan des 5.-6. Jahrhundert n. Chr. schließlich wohl denkbar wäre. In dieser Zeit wurde der Name aber sicherlich als (substantiviertes)

(2012: 38) die einzige bekannte (und zugegebenermaßen unsichere) Instanz „de la commémoration des parents par alliance" wäre.

Die Identifizierung der geeigneten Heiligen, die als Auslöser für die entsprechenden christlichen $\operatorname{cog}$ nomina des Iustinos gedient haben können, ist sicherlich nicht einfach und muss unsicher bleiben. Wenn man aber seinen Vater Germanos, den zweimaligen magister militum und im allgemeinen einen berühmten Heerführer, der zwischen 536 und 539 in Afrika gewirkt hat (s. zu ihm PLRE II: 505-507 [s. v. Germanus 4]), als den eigentlichen Namengeber vermuten würde, könnte man an den Soldatenheiligen Theodoros „Tiro“ (zu ihm s. Delehaye [1909: 11-26], Oikonomides [1986: 327-329] und Walter [1999: besonders 170-172]) und an zwei Märtyrer namens Valentinus und Rusticus denken, die offenbar auch mit Afrika verbunden waren (Valentinus: AASS Febr. II: 763; Rusticus: AASS Mar. I: 365-366). „Theodoros“ wird so auch von Laniado (2012: 42 und 47) als devotionales cognomen in einigen anderen polyonymen Reihen identifiziert und mit dem Soldatenheiligen verknüpft.

57 Stein (1949: 275f.).

58 Leppin (2011: 30). Der Forscher relativiert seine Hypothese aber selbst (der Name als Familientradition).

$59 \mathrm{Zu}$ den Varianten des Namens der Gottheit s. Eisele (1909-1915: 232-233). 
Adjektiv zum jüdischen und folglich auch christlichen Begriff des Sabbat verstanden ${ }^{60} \mathrm{Im}$ Lichte der offenbar unlateinischen Namen von Iustinos' angeblichen ehemaligen Gefähr-

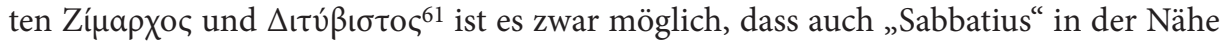
von Bederiana letzten Endes heidnischen oder wenigstens sprachlich etwa thrakischen ${ }^{62}$ Ursprungs war, es ist aber höchst unwahrscheinlich, dass man sich dessen während Iustinianos' Lebens noch bewusst war.

Der wahre Grund dessen, dass sich Iustinianos als Kaiser nicht mehr Petros Iustinianos oder sogar Petros Sabbatios Iustinianos nannte, ist meiner Meinung nach darin $\mathrm{zu}$ suchen, dass die römischen Kaiser vor ihm, zumindest die aus Konstantinopel herrschenden, vom gentilicium Flavius abgesehen, das eher selten und vorwiegend epigraphisch bezeugt ist, ${ }^{63}$ seit Iovianus (363-364) bzw. bereits seit Constantius II. (337-361) nur einen Namen getragen hatten. ${ }^{64}$ Während also Iustinianos noch als Mittglied der

60 Zum „Christian calendaric cognomen“ Sabbatius vgl. Kajanto (1965: 62 und 135). Im 5. Jahrhundert findet man in Konstantinopel zwei Männer dieses Namens, einen von ihnen angeblich jüdischer Herkunft: Socr. Hist. eccl. V, 21, 6 und VII, 30, 7.

61 Sie werden genannt bei Procop. Arc. 6, 2. Wie mir allerdings scheint, kann man Zímapxoৎ als griechi-

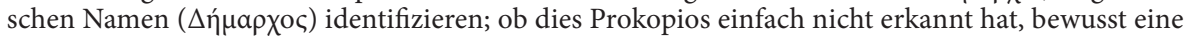
abweichende Aussprache des anlautenden Frikativs reflektierte bzw. konstruierte (und dabei etwa Iustinos und seine Gefährten implizit als barbarische Personen verhöhnte) oder aber hier vielleicht sogar einen intertextuellen Verweis in die Anekdota verbaute (dann am ehesten an Thuc. VIII, 85, 3 und Xenoph. Hell. I, 1, 29; allerdings vermöchte ich dann seine Botschaft nicht recht zu verstehen), mag dahingestellt bleiben. Der Name ist jedenfalls im 6. Jahrhundert unter den Angehörigen der Elite und Subelite des Reiches nicht ganz selten (s. PLRE II: 1196 [s.v. Zemarchus] und PLRE III: 1416-1417 [s.v. Zemarchus 1-5]); und obwohl dieser oder jener Würdenträger ein Verwandter oder Nachkomme des Gefährten von Iustinos gewesen sein konnte, ist es jedoch unwahrscheinlich, dass alle diese Männer einen Namen thrakischen Ursprungs getragen haben: zwei unter ihnen sind in Italien bezeugt (PLRE III: 1417 [s.v. Zemarchus 4-5]) und der Abgesandte zu den Türken im Jahre 569 soll „Kiliker“ gewesen sein (Men. Prot. fr. 10, 2 [Blockley]). Immerhin ist es mir nicht gelungen, auch für $\Delta$ เ úßı

62 Wie man mindestens seit Roesler (1873: 116) zu behaupten pflegt.

63 S. Rösch (1978: 49-50), der allerdings seine nicht-epigraphischen Belege Nr. 34 und 42 unberücksichtigt lässt.

64 Vgl. die Materialsammlung bei Rösch (1978: 159-167). Die einzige Ausnahme ist hier Valentinianus III., also ein westlicher Kaiser, mit einem weiteren cognomen zur Ehre seiner Mutter, das die berühmte Inschrift CIL VI 1783, Z. 7-8 bezeugt: IMPERATORES CAESS. FL THEODOSIVS ET FL PLACIDVS valentinianvs SEmper AVGg. Als den Autor dieses Textes hatte Hedrick (2000: 224-225) Nicomachus Flavianus den Jüngeren vorgeschlagen, was etwa mein Kollege I. Prchlík (2015: 15 mit Anm. 38) - ohne die bei ihm sonst übliche eingehende Diskussion - als „a very persuasive argument“ akzeptierte. Ich persönlich halte das für eine denkbare und im gewissen Sinne sogar wahrscheinliche Hypothese, nicht aber für eine glücklich formulierte und beinahe gesicherte Tatsache. Wie nämlich Hedrick (2000: 224) selbst einräumt, wurden die kaiserlichen Schreiben von den zugehörigen Beamten, also im Fall eines Briefes von solcher (politischen) Bedeutung vermutlich vom quaestor sacri palatii verfasst bzw. diktiert (vgl. z.B. Delmaire 1995: 59-61). Nicomachus Flavianus, der damalige praefectus praetorio Italiae, Illyrici et Africae (s. zu ihm PLRE I: 345-347 [s.v. Flavianus 14]), war aber keineswegs Vorgesetzter dieses Beamten bzw. Ministers; und dass der uns namentlich unbekannte quaestor "a friend or dependent" von Flavianus gewesen sein mag (Hedrick 2000: 224), muss eine bloße Spekulation bleiben. Da es weiter kaum denkbar ist, dass Flavianus den quaestor beim Verfassen des kaiserlichen Schreibens umgehen konnte oder auch nur wollte (was doch die Ordnungsmäßigkeit der Rehabilitation seines Vaters beeinträchtigen konnte!), kann man zwar etwa die Hypothese formulieren, dass er dem quaestor gegenüber seine Vorstellungen über den Inhalt des Briefes geäußert oder aber ihm sogar eine schriftliche Vorlage geliefert hatte (in diesem Sinne Cameron 2011: 205), weder kann man aber eine solche Vermutung beweisen noch kann man wissen, inwieweit jene eventuelle Vorlage beim Verfassen des Schreibens wirklich berücksichtigt wurde. Und auch wenn Nicomachus Flavianus der faktische Autor des Schreibens in vollem Umfang gewesen wäre, als der offizielle Autor muss der quaestor sacri palatii gegolten haben, von dem auch - und das ist hier das 
östlichen Eliten konform zu ihrem Gebrauch der polyonymen Namenreihen gewesen war, ${ }^{65}$ hatte er als Kaiser keinen Anlass mehr, mit zwei oder drei cognomina zu prunken, sondern war vielmehr von der Tradition zum einfachen Namen geleitet. Sicherlich kann man zwar behaupten, dass er nicht strikt daran gebunden war, sich dieser Protokollgewohnheit zu fügen. Die Unbekümmertheit, mit der er dies aber getan hat, spricht somit dafür, dass Petros und Sabbatios nichts mehr als sekundäre cognomina zum eigentlichen und ursprünglichen Namen Iustinianos waren.

Wenn man Iustinianos' Adoption als Tatsache akzeptiert, und zwar als Vorgang aus der Zeit der Anfänge von Iustinos' Herrschaft zwischen den Jahren 518 und 520 (was unsicher bleiben muss, da eine noch frühere Datierung nicht auszuschließen ist), scheint mir die Ablehnung der Auffassung, dass sich der Kaiser diesen Neffen als seinen Nachfol-

Entscheidende - der offizielle Charakter des Textes garantiert wurde. Man muss also damit rechnen, dass Valentinianus III. als Kaiser das cognomen Placidus im Jahre 431 in den offiziellen Akten wirklich getragen hat - eine Ausnahmeerscheinung, die auf die politische Bedeutung seiner Mutter Galla Placidia zurückzuführen ist.

Eine Inschrift aus Delphi, zuletzt ediert von Vatin (1962: 238), nennt die Kaiser Valentinianus I.

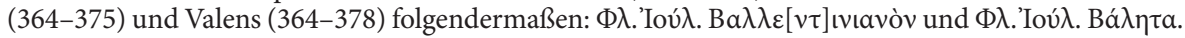
Laniado (2012: 27 mit Anm. 2) lässt deshalb die Serie der östlichen Kaiser, die keine polyonyme Reihe getragen haben, erst mit Valens' Nachfolger Theodosius I. zu beginnen. Dazu sehe ich keinen schwerwiegenden Grund: die Inschrift stammt natürlich nicht aus der kaiserlichen Kanzlei und bietet uns folglich so gut wie keine Garantie, dass einer oder beide der Kaiser sich selbst so bezeichnet haben; einer solchen Annahme widersprechen auch die Quellen der offiziellen Provenienz (gesammelt bei Rösch 1978: 162). Es handelt sich wahrscheinlich um einen Irrtum des Stifters der Inschrift, der delphischen Polis (in diesem Sinne bereits Vatin 1962: 238), inspiriert vielleicht vom Vollnamen des Kaisers Constantius II. (337-361).

Die polyonymen Namen sind für die Kaiser der konstantinischen Dynastie gut bezeugt; im Osten haben Constantinus I. (Fl. Val. Constantinus; zu den Belegen s. PLRE I: 223 [s.v. Constantinus 4]), der eben genannte Constantius II. (Fl. Iul. Constantius; PLRE I: 226 [s.v. Constantius 8]) sowie Iulianus (Fl. Cl[audius] Iulianus; PLRE I: 477 [s.v. Iulianus 29]) geherrscht. Zu beachten ist allerdings, dass diese polyonymen Namen anders als jene des 5.-6. Jahrhunderts gestaltet sind (Valerius, Iulius und Claudius sind ähnlich wie Flavius nomina, nicht cognomina) und schon deshalb für Iustinianos als Kaiser keine besonders geeignete Vorbilder sein konnten. Noch wichtiger ist, dass man die Belege für „Fl. Iul. Constantius“ (als Augustus) und „Fl. Claudius Iulianus“ nicht in den Schreiben aus der kaiserlichen Kanzlei (abermals sei auf das Material bei Rösch [1978: 159-161] verwiesen), sondern nur in den Inschriften privater Personen und allenfalls auf den Münzen findet. Zwischen den Jahren 337 und 363 haben also, soweit man sehen kann, die Kaiser polyonyme Namen zwar immer noch gehabt, sie jedoch nicht in den offiziellen Schriftstücken, also bei der feierlichen Selbstpräsentation als Herrscher, benutzt.

Der Fall von Constantinus I. ist nicht ganz eindeutig. Als der seit dem Jahre 324 einzige Augustus erscheint er in seinen Schreiben unter dem einfachen Namen, mit der Ausnahme der Inschrift CIL XI 5265 , Z. 2-4. Es ist möglich, dass er hier die polyonyme Reihe FL CONSTANTINvs trägt, um sich seinen drei Söhnen in dieser Hinsicht anzugleichen, die, obwohl Caesares, hier (CIL XI 5265, Z. 4-6) ohne Titel genannt sind. Anders in CIL III 7000, III, Z. 4-7: Fl. Claudius Constantinus und Fl. Iulius Constantius werden nicht nur mit ihren polyonymen Namen genannt, sondern auch tituliert, ihr Vater trägt hier aber kein nomen (gentilicium). Bereits unter Constantinus I. bildet sich also anscheinend das Bewusstsein, dass der polyonyme Name für den Kaiser bei der Selbstpräsentation als Herrscher eher unpassend ist, sofern er ihn nicht als Mitglied einer Familie bezeichnen oder aber als Person identifizieren soll; es ist also vermutlich symptomatisch, dass Constantinus' Söhne ihre polyonymen Reihen in den Schriftstücken nicht mehr benutzen, sobald sie Augusti geworden sind.

65 Das Material für die östlichen Konsuln bzw. Inschriften aus ihren Diptychen erwähnt in aller Kürze etwa Al. Cameron (1988: 31). 
ger schon damals ausgewählt hat, wenig sinnvoll, ${ }^{66}$ es sei denn, man würde die Adoption als eher widerwillig ansehen. ${ }^{67}$

Natürlich wurde Iustinianos dadurch offiziell noch kein Thronfolger, ${ }^{68}$ das brauchte ihn aber wenig zu stören. Wichtiger musste für ihn sein, sich die Akzeptanz auch bei anderen als beim Adoptivvater zu sichern, die nach dem Tod des Letztgenannten über den neuen Kaiser entscheiden könnten. Lediglich weitere Verwandtschaft zum Vorgänger war nämlich, wie die Praxis gezeigt hatte, noch keine Garantie für die Nachfolge, ${ }^{69}$ und die Adoption seitens des Kaisers war am Anfang des 6. Jahrhunderts ein seit Jahrzehnten, ja Jahrhunderten vergessenes und somit in ihrer Wirkung unerprobtes Mittel zur Sicherung des Thrones..$^{70}$ Es gibt Anzeichen, dass Iustinianos in dieser Hinsicht tätig war: neben der uns bereits bekannten vermutlichen Bemühung, als Anhänger des Papsttums und des Konzils von 451 die Popularität bei den dezidierten Chalkedonianern zu gewinnen, war Iustinianos’ „Inszenierung als Consul“ des Jahres 521 mit den glänzenden Spielen „bereits die Inszenierung eines künftigen Herrschers" ${ }^{\text {"71 }}$ vor allem, wenn auch sicherlich nicht ausschließlich, gegenüber dem „Volk“ als einer der Akzeptanzgruppen.

66 Vgl. die Analysen von Croke (2007) und Leppin (2011: 73-75 und 89). Es wird von ihnen u.a. betont, dass Iustinos mit Iustinianos' Förderung gezögert hat. Das ist im Ganzen genommen unsicher. In den Quellen wird soweit nur von Iustinianos' Ernennung zum nobilissimus behauptet, wobei es sich allerdings um eine späte und vor allem anekdotisch gefärbte Schilderung handelt (Ioh. Zonar. Epit. hist. XIV, 5, 35-37). Für die Caesar-Würde postuliert Croke (2007: 44) ähnliches aufgrund Vict. Tonn. Chron. ad a. 525 (Iustinus Augustus Iustinianum nepotem suum ad senatorum supplicationem invitus Caesarem facit), dessen unklarer Text aber auch für Iustinos' Initiative sprechen kann: Iustinos soll streng genommen zur supplicatio, nicht zur Caesar-Ausrufung eingeladen worden sein und diese supplicatio kann nicht eine Bitte, etwa um diesen Akt, sondern nur eine festliche Akklamation sein, so dass der Kaiser das Programm sozusagen geändert oder ergänzt hat. Schließlich ist Noethlichs' (2001: 675) Vermutung, die Erhebung des Iustinianos zum Augustus sei keine Initiative des Iustinos gewesen, was sogar eher gegen die Adoption spreche, auf problematischer Grundlage gegründet: in Procop. Arc. 9, 52 sind es im Gegenteil die Senatoren, nicht Iustinos, die widerwillig den neuen Kaiser akklamieren, und die eben erwähnte Stelle Victors von Tunnuna gehört nicht hierher, wenigsten im Sinne der Rekonstruktion von Vasiliev (1950: 94-95 mit Anm. 70) und Croke (2007: 44-45 mit Anm. 166 und 167); anders neuerdings Szidat (2014: 881 Anm. 13).

Auch wenn man Iustinos' Zögern als Tatsache akzeptieren würde, würde sie a priori nur dafür sprechen, dass er den Neffen und Sohn von der Machtausübung bzw. von der Berechtigung zu ihr fern halten wollte (in diesem Sinne auch die erste von Leppin [2011: 89] erwogene Möglichkeit: „Musste der alte Herr nicht Angst haben, dass sein Neffe ihn an den Rand drängen werde?"). Dafür aber, dass er nach der Adoption noch Alternativen zu Iustinianos als Nachfolger erwog und aufbaute - vgl. Leppin (2011: 73, auch 89 [„Wollte er die angenehme Situation genießen, gegebenfalls andere Persönlichkeiten ... gegen Justinian auszuspielen?"]) -, gibt es, soweit ich sehe, keine ernsthafte positive Indizien.

67 Dem nähert sich die Überlegung von Croke (2007: 21): „Indeed, it may have been a response to those who had been prepared to nominate him as emperor ahead of his uncle in July 518." Die Nachricht des Const. Porph. De cer. I, 93 (428 Reiske), Iustinianos sei nach dem Tod des Anastasios von allen excubitores aufgefordert worden, die Kaiserwürde anzunehmen, ist aber nicht über jeden Zweifel erhaben; vgl. Noethlichs (2001: 675) sowie Pfeilschifter (2013: 170), der sich jedoch für die Historizität der Nachricht entscheidet.

68 Das bedeutete nach Croke (2007: 44 und 46) erst der ihm im Jahre 525 erteilte Caesar-Titel.

69 Eine sehr frische und persönliche Erfahrung dieser Tatsache hat Iustinianos das Übergehen von Neffen des Anastasios bei der Wahl des neuen Kaisers im Jahre 518 geboten; vgl. dazu Pfeilschifter (2013: 149-150, 165-172 und 175-176).

70 Vgl. etwa den Überblick von Pfeilschifter (2013: 125-130, 137-140 und 147-150). Zeitlich am nächsten stand den Vorgängen in der Periode von Iustinos und Iustinianos die tetrarchische Praxis der Adoption der Caesares an der Wende vom 3. zum 4. Jahrhundert; zu ihr s. Kolb (1987: 8-9, 68-69 und 178).

71 So Leppin (2011: 74). Die Spiele sind bei Marcell. Chron. ad a. 521 beschrieben. 
Man kann sich also fragen, ob Iustinianos dabei nicht auch ein geschichtliches Thema aufgegriffen hat, nämlich die vielleicht überhaupt berühmteste und folgenreichste Adoption der römischen Geschichte. Hinweise darauf, dass am Anfang der Karriere C. Octavius', des späteren Kaisers Augustus, die Adoption durch seinen Großonkel C. Iulius Caesar stand, konnten die Bekanntheit und somit Bedeutung dieses Akts als längst vergessenen Mittels zur Sicherung der Thronfolge nur erhöhen und die mit der jeweiligen Adoption verbundene Analogie zwischen dem ersten römischen Kaiser und Iustinianos das Bild des Letztgenannten vervollkommnen.

Dass im Zusammenhang mit der Adoption der Kaiser Augustus heute ein sehr naheliegendes Beispiel aus der römischen Geschichte ist, zeigt in aller Anschaulichkeit etwa die bereits zitierte, wenn auch nicht ganz richtige, Erörterung von Croke. ${ }^{72}$ Dass dies Iustinianos bewusst sein und seiner Propaganda gedient haben kann, scheint mir aus folgenden Gründen denkbar: obwohl die Erudition von Ioannes Lydos sicherlich außergewöhnlich war, bezeugt seine Ausführung über Augustus ${ }^{73}$ vielleicht doch das einigermaßen vorhandene Bewusstsein von diesem Kaiser in denjenigen Kreisen, die hinreichende Ausbildung besaßen, „the conservative, classically trained aristocrats and the bureaucratic "sub-elite" " ${ }^{74}$ Zweitens sieht man in Iustinianos' Novellen aus den 30er Jahren des 6. Jahrhunderts, dass die historische (oder vielmehr pseudohistorische) Argumentation eine sehr prominente Rolle spielt, vielleicht eben mit Rücksicht auf jene klassisch gebildeten Kreise. ${ }^{75}$ Weiter ist zu beachten, dass Marcellinus Comes, dessen Nähe

72 Vgl. oben S. 206. Da Iustinianos sowohl vor als auch nach der Adoption immer so geheißen hat, entfällt die Möglichkeit, die Parallele zwischen dem Erbfolger Caesars und jenem des Iustinos auch in der Tatsache der Namensänderung zu sehen. Immerhin vermag das Suffix -ianus bzw. -ianos, wenn Iustinianos und Octavianus einmal in Verbindung gebracht werden, offensichtlich, beide Namen weiter zu assoziieren. Vielleicht konnte es so auch im 6. Jahrhundert wirken, obwohl die cognomina auf -ianus bzw. -ianos in der spätantiken Zeit durchaus üblich waren und manche ihrer Träger (Diocletianus, Vitalianus) Iustinianos' Bewusstsein und Propaganda unbequem sein mussten. Die Frage, ob der unter dem Namen C. Iulius Caesar adoptierte Octavius jemals selbst das cognomen Octavianus getragen hat, kann auf sich beruhen (traditionell lautet die Antwort negativ, so z.B. bei Kienast [1996: 61]; Shackleton-Bailey [1976: 93] bleibt jedoch vorsichtiger). In der späteren Überlieferung war er jedenfalls auch unter der Bezeichnung „Octavianus“ bekannt.

73 Ioh. Lyd. Mag. II, 3. Ob dabei Lydos gegenüber Augustus kritisch ist und damit eine Kritik an Iustinianos und überhaupt am Kaisertum beabsichtigt, wie Kaldellis (2005: besonders 6-8 und 11) meint, mag in diesem Zusammenhang dahingestellt bleiben. Die Adoption wird konkret in Ioh. Lyd. Mag.

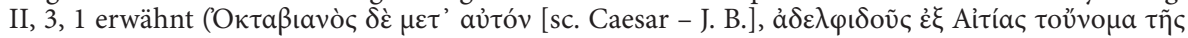

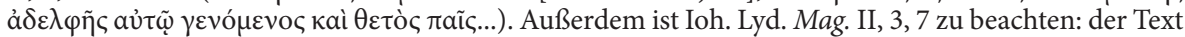

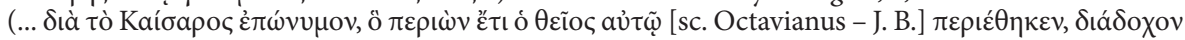

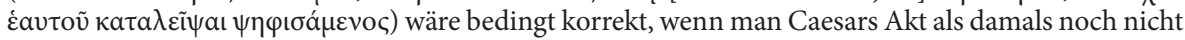
bekannt bzw. sein Testament als noch unveröffentlicht verstünde, doch erweckt der Abschnitt eher den Eindruck, dass Caesar seinen „Nachfolger" ganz öffentlich verkündete. Sollte man hier vielleicht eine Anspielung an (vgl. Schamp 2006: CCCLXIII [„Un lecteur du VIe s. devait songer aux conditions de succession au trône à son époque.“]) oder eine Spitze gegen Iustinos' Adoption des Neffen vermuten? Sicherlich falsch ist die Präsentation des genealogischen Verhältnisses zwischen Caesar und Octavianus in Ioh. Lyd. Mag. II, 3, 1, die aber jenem zwischen Iustinos und Iustinianos entspricht. Haben wir hier einen bloßen Irrtum oder aber die absichtliche Verzerrung der Fakten vor uns? In Ioh.

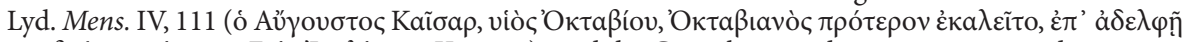

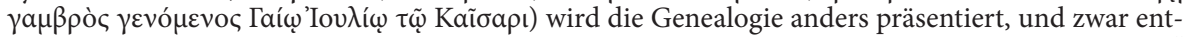

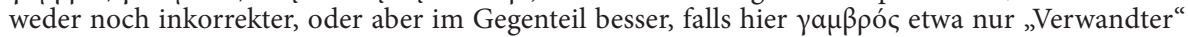
bedeuten sollte.

74 Diese Terminologie übernehme ich von Maas (1986: 25f.).

75 S. dazu Maas (1986: 18-19 und 24-26). Der Forscher neigt dazu, die Propaganda, deren Mittel die Novellen waren, als Reaktion auf den Nika-Aufstand aufzufassen und als ihr Ziel die provinzialen 
zu Iustinianos zuweilen zwar übertrieben wird, der allerdings für Iustinianos vor dem Jahre 525 oder 527 als Kanzler gearbeitet hat, ${ }^{76}$ in seiner Chronik Augustus kennt, ${ }^{77}$ so dass man Entsprechendes auch für den späteren Kaiser voraussetzen darf.

Man muss zugeben, dass diese Indizien für die Bekanntheit von Octavianus Augustus im Ganzen genommen eher für die Zeit von Iustinianos' Regierung relevant sind, ${ }^{78}$ doch wäre vermutlich akzeptabel, aus ihnen entsprechende Schlüsse auch für die 20er Jahre zu ziehen. Die Persönlichkeit des ersten Kaisers scheint zunächst nur in Hinsicht auf die konstantinopolitanische Elite und Subelite ein geeignetes Mittel der Propaganda zu sein; doch das können eben die Gruppen sein, auf die Iustinianos zielen wollte, insofern das Volk etwa mit den Spielen und der Treue zum Konzil von Chalkedon sozusagen schon bedient wurde.

Da wir keine Spuren einer solchen mit Augustus arbeitenden Propaganda aus Iustinos' Regierung haben und angesichts der weitgehend fehlenden passenden Quellen aus dieser Zeit sie auch nicht haben können, müssen diese Überlegungen theoretisch bleiben. Trotzdem gibt es wenigsten einige Texte, zu deren Analyse die Hypothese aufschlussreich zu sein scheint, dass Iustinianos seiner Adoption durchaus Bedeutung beigemessen hat; ${ }^{79}$ und die bereits besprochenen Stellen von Ioannes Lydos legen sogar auch nahe, dass der Fall des Augustus in der damaligen mit der Thronfolgeregelung durch die Adoption operierenden Propaganda, wenn nicht sogar im politischen Diskurs, eine Rolle gespielt hat. ${ }^{80}$

Hinweisen möchte ich noch auf die Nachricht des Prokopios von Kaisareia über die zuletzt erfolglose Bestrebung des persischen Königs Kabades I., seinen Sohn und späteren Nachfolger Chosroes von Kaiser Iustinos adoptieren zu lassen. ${ }^{81}$ Die Historizität der Geschichte bzw. ihres Kerns wurde bisher nicht bezweifelt, obwohl man, wie mir scheint, die Möglichkeit ernst nehmen muss, dass sie von Prokopios selbst erfunden wur-

Aristokraten zu sehen. Beides würde aber nicht ausschließen, dass Iustinianos das gleiche Mittel schon früher mit Hinsicht auf die konstantinopolitanische Elite und Subelite ausprobiert hatte. Kruse (2015: 233) seinerseits glaubt, dass unter den Lesern der einschlägigen Novellen auch die (in Konstantinopel lebenden) ,ambitious members of the senate“ sowie „numerous bureacrats and secretaries such as John Lydus and Procopius" waren.

76 Cassiod. Inst. I, 17, 2 (patricii Iustiniani fertur egisse cancellos). Zur Datierung s. Croke (2001: 28).

77 Und zwar im überhaupt bekanntesten Lemma des Werkes über die Absetzung des Romulus Augustulus (Marcell. Chron. ad a. 476, 2): Hesperium Romanae gentis imperium, quod ... primus Augustorum Octavianus Augustus tenere coepit, ... periit... Zu diesem Eintrag vgl. etwa Goltz (2008: 95-103) und Jakobi (2011), beide mit früherer Literatur.

78 Eine Ausnahme wäre die Chronik von Marcellinus Comes, sollte man mit der Forschung ihre erste Redaktion bereits vor Iustinianos' Kaisertum datieren; s. Croke (2001: 26-28) sowie - besser - Goltz (2008: 105-114). Das halte ich für eigentlich unbegründet, auf die Problematik hoffe ich aber anderswo einzugehen.

79 Das Gegenteil hat Noethlichs (2001: 675) behauptet: „Zur Legitimation seiner Herrschaft ... spielte für I. jedenfalls die Adoption keine Rolle." Es wäre nicht überraschend, dass sich Iustinianos nicht mehr als Iustinos' Sohn und Erbe präsentierte, nachdem er sich die Regierung bereits gesichert hatte; von der Zeit davor sagt das bei der Absenz der Quellen wenig aus. In einem offiziellen Text aus dem Jahre 535 ist immerhin eine Aussage enthalten, die Gott als die eigentliche Quelle der Herrschaft des Iustinianos nennt, daneben auch auf die angebliche allgemeine Wahl Bezug nimmt (was die These von Meier [2003a: 118 und 122-123], Iustinianos „fühlte sich nur noch von Gott eingesetzt, nicht aber mehr vom Volk bestimmt", zumindest einigermaßen relativiert) sowie den Kaiser als Erben seines Vaters

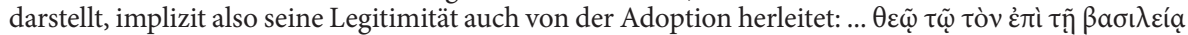

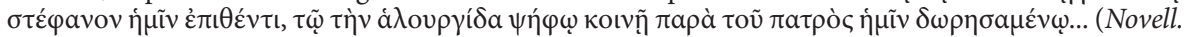
Iust. 28, 4, 2).

80 Oben Anm. 73.

81 Procop. Pers. I, 11. 
de. ${ }^{82}$ Wie dem aber auch sein mag, der Historiker von Kaisareia hat die Darstellung für seine Ziele benutzt, ${ }^{83}$ nämlich für die diskrete Kritik an Iustinianos: Der quaestor sacri palatii Proklos wird hier als positiver Gegensatz zu Iustinianos und seinen Schützlingen geschildert. ${ }^{84}$

Man kann aber bei einer solchen Interpretation noch weiter gehen und sich fragen, warum Prokopios einen seiner Helden gerade an dieser Stelle des Werkes vorführt. Dieser figuriert hier als Kritiker des angeblichen Vorschlags der Adoption des persischen Prinzen. Soll er also eigentlich die Kritik einer anderen Adoption bzw. ihrer Folgen artikulieren? Es ist vermutlich weder Zufall noch ohne Bedeutung, dass Iustinianos, der in diesem Zusammenhang in Prokopios' Kriegen zum ersten Mal auftritt und, wenn man vom Prooimion absieht, erstmals überhaupt genannt wird, hier zwar als Iustinos' Neffe sowie erwarteter Thronfolger vorgestellt wird, von der relevanten Tatsache seiner Adoption aber geschwiegen wird: ${ }^{85}$ vielleicht sollte das einerseits dem Autor ein Alibi sichern vor der Beschuldigung, dass er beide Adoptionen vergleichen wolle, andererseits aber die das auffällige Schweigen bemerkenden Leser auf die Möglichkeit dieses Vergleichs aufmerksam machen.

Die bei Prokopios überlieferte Rede des Proklos über die eventuellen Folgen der Adoption kann man interpretierend folgendermaßen zusammenfassen: Des Reiches würden sich Barbaren bemächtigen, Iustinos könnte der letzte römische Kaiser sein. ${ }^{86}$ Liest man diese Präsentation im Lichte dessen, was man in Prokopios' Anekdota findet, ${ }^{87}$ fällt es nicht schwer, sie zur Klage über den dank der Adoption ermöglichten Herrschaftsantritt des Iustinianos umzudeuten. Vielleicht ist darin auch der Vorwurf an die Eliten enthalten, dass sie Iustinianos' Machtübernahme nicht verhindert haben. Jedenfalls ist sie bedeutsam als Indiz der Wichtigkeit, die Prokopios der fraglichen Adoption beigemessen hat und die der Akt auch für Iustinianos und seine Propaganda wahrscheinlich einst gehabt hatte.

82 Zur communis opinio vgl. Börm (2007: 311-317) und Brandes (2013: 266-267), beide mit früherer Literatur.

Ausschlaggebend scheint mir zu sein, dass Prokopios für uns eigentlich die einzige unabhängige Quelle ist. Die anderen Nachrichten sind entweder ausdrücklich von ihm exzerpiert bzw. nennen ihn als Quelle (Euagr. Hist. eccl. IV, 12; Phot. Bibl. LXIII, 23a, 13-27 [Henry]; Const. Porph. Exc. de leg. I, 90-91 [de Boor]; Niceph. Callist. Xanthopul., Hist. eccl. XVII, 10 [eigentlich nach Euagrios]), oder aber es besteht kein Anlass, Prokopios als ihre direkte oder indirekte Quelle zu bestreiten (Theoph. Chron. ad a. 6013 [167-168 de Boor]; Ioh. Zonar. Epit. hist. XIV, 5, 22; Theophanes' Angabe, dass

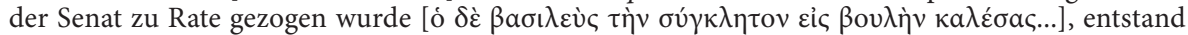

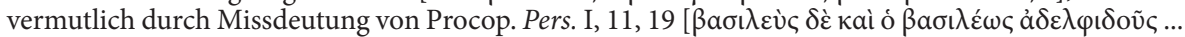

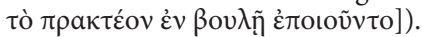

Wieso man sich dann der Historizität dieser Geschichte sicher sein soll, wenn sogar zugegeben wird, dass sie in Prokopios' Schilderung Unklares oder Problematisches enthält (vgl. Börm 2007: 312 und 314-316), leuchtet mir nicht ein.

83 Für den Anfang von Prokopios' Werk Kriege im Allgemeinen hat das Kaldellis (2004: 62-93) zu zeigen versucht; darunter zu Kabades' Thronfolgeplänen Kaldellis (2004: 85-86).

${ }^{84}$ Signes Codoñer (2003: 221).

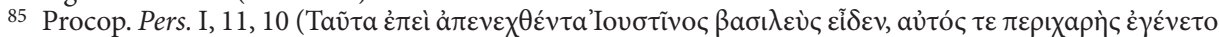

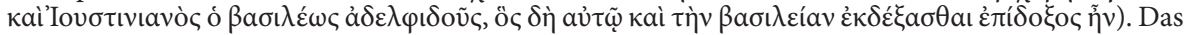

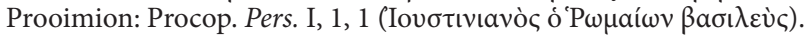

86 Procop. Pers. I, 11, 13-18 (daneben noch I, 11, 21-22).

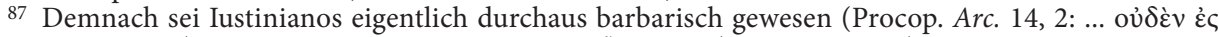

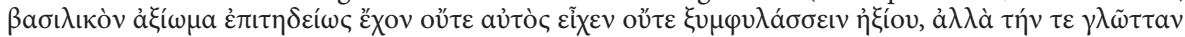

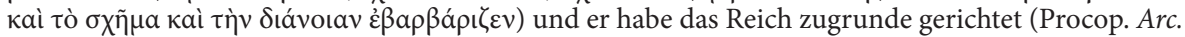

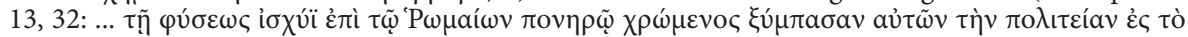

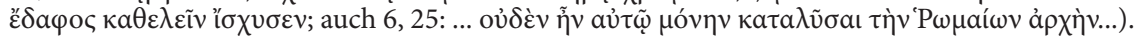




\section{BIBLIOGRAPHIE}

AASS $=$ Acta sanctorum $.1643-$.

ACO III = Collectio Sabbaitica contra acephalos et origeniastas destinata. Insunt acta synodorum Constantinopolitanae et Hierosolymitanae a. 536. Ed. E. Schwartz [= Acta conciliorum oecumenicorum. Tomus tertius]. Berolini: Walter de Gruyter, 1940 (Nachdruck 1965).

ACO IV/1 = Concilium universale Constantinopolitanum sub Iustiniano habitum. Volumen primum. Concilii actiones VIII. Appendices Graecae. Indices. Ed. J. Straub [= Acta conciliorum oecumenicorum. Tomus quartus. Volumen primum]. Berolini: Walter de Gruyter, 1971.

Antès, S., 1981. Corippe (Flavius Cresconius Corippus), Élogé de l'empereur Justin II. Texte établi et traduit par S. Antès. Paris: Société d'édition Les belles lettres.

Börm, H., 2007. Prokop und die Perser. Untersuchungen zu den römisch-sasanidischen Kontakten in der ausgehenden Spätantike. Stuttgart: Franz Steiner Verlag.

Brandes, W., 2013. „Die »Familie der Könige« im Mittelalter. Ein Diskussionsbeitrag zur Kritik eines vermeintlichen Erkenntnismodells“. Rechtsgeschichte 21, 262-284.

Bury, J. B., 1923. History of the Later Roman Empire from the Death of Theodosius I to the Death of Justinian. Volume II. Repr. New York: Dover Publications, 1958.

Cameron, Al., 1976. „Theodorus triseparchos“. Greek, Roman and Byzantine Studies 17, 269-286 = Al. Cameron, Literature and Society in the Early Byzantine World. London: Variorum Reprints, 1985, Nr. XVI.

Cameron, Al., 1985. „Polyonymy in the Late Roman Aristocracy: the Case of Petronius Probus“. The Journal of Roman Studies 75, 164-182.

Cameron, Al., 1988. „Flavius: a Nicety of Protocol“. Latomus 47, 26-33.

Cameron, Al., 2011. The Last Pagans of Rome. Oxford [et al.]: Oxford University Press.

Cameron, Av., 1975. „The Empress Sophia“. Byzantion 45, 5-21 = Av. Cameron, Continuity and Change in Sixth-Century Byzantium. London: Variorum Reprints, 1981, Nr. XI.

Cameron, Av., 1976. Flavius Cresconius Corippus, In laudem Iustini Augusti minoris. Ed. with translation and commentary by Av. Cameron. London: University of London, The Athlone Press.

CIL $=$ Corpus inscriptionum Latinarum. Berlin, 1862-.

Croke, B., 2001. Count Marcellinus and his Chronicle. Oxford: Oxford University Press.

Croke, B., 2007. „Justinian under Justin: Reconfiguring a Reign“. Byzantinische Zeitschrift 100, 13-56.

Delehaye, H., 1909. Les légendes grecques des saints militaires. Paris: Librairie Alphonse Picard et fils.

Delmaire, R., 1995. Les institutions du Bas-Empire romain, de Constantin à Justinien. I. Les institutions civiles palatines. Paris: Les Éditions du CERF / Les Éditions du CNRS.

Eisele, Th., 1909-1915. „Sabazios“. In: W. H. Roscher (ed.), Ausführliches Lexikon der griechischen und römischen Mythologie. Vierter Band. Qu-S. Leipzig: B. G. Teubner, 232-264.

Evans, J. A. S., 1996. The Age of Justinian. The circumstances of imperial power. London / New York: Routledge.

Feissel, D., Kaygusuz, I., 1985. „Un mandement impérial du VIe siècle dans une inscription d'Hadrianoupolis d'Honoriade“. In: Feissel (2010: 223-250) [ursprünglich: Collège de France. Centre de recherche d'histoire et civilisation de Byzance. Travaux et mémoires 9, 397-419].

Feissel, D., 2004. „Un rescrit de Justinien découvert à Didymes (1 ${ }^{\mathrm{er}}$ avril 533)“. In: Feissel (2010: 251-324) [ursprünglich: Chiron 34, 285-365].

Feissel, D., 2010. Documents, droit, diplomatique de l'Empire romain tardif. Paris: Association des amis du Centre d'histoire et civilisation de Byzance.

Gascou, J., 2002. „Décision de Cesarius, gouverneur militaire de Thébaide“. In: J. Gascou, Fiscalité et société en Égypte byzantine. Paris: Association des amis du Centre d'histoire et civilisation de Byzance, 431-439 [ursprünglich: Collège de France. Centre de recherche d'histoire et civilisation de Byzance. Travaux et mémoires 14, 269-277].

Gignac, F. Th., 1981. A Grammar of the Greek Papyri of the Roman and Byzantine Periods. Volume II. Morphology. Milano: Istituto Editoriale Cisalpino - La Goliardica.

Goltz, A., 2008. Barbar - König - Tyrann. Das Bild Theoderichs des Großen in der Überlieferung des 5. bis 9. Jahrhunderts. Berlin / New York: Walter de Gruyter. 
Greatrex, G., 2007. „The early years of Justin I’s reign in the sources“. In: E. Dąbrowa (ed.), Studies on the Late Roman History [= Electrum 12]. Kraków: Jagiellonian University Press, 99-113.

Guenther, O., 1895-1898. Epistulae imperatorum pontificum aliorum inde ab a. CCCLXVII usque ad a. DLIII datae. Avellana quae dicitur collectio. Pars I-Pars II. Recensuit commentario critico instruxit indices adiecit O. Guenther [= Corpus scriptorum ecclesiasticorum Latinorum. Vol. XXXV]. Pragae / Vindobonae / Lipsiae: F. Tempsky / G. Freytag.

Hedrick, Ch. W., 2000. History and Silence. Purge and Rehabilitation of Memory in Late Antiquity. Austin: University of Texas Press.

Holmes, W. G., 1912. The Age of Justinian and Theodora. A History of the Sixth Century A.D. Vol. I. Second ed. London: G. Bell and sons.

Jakobi, R., 2011. „Marcellinus Comes über das Ende des Kaisertums im Westen des römischen Reiches. Eine quellenkritische Petitesse“. Philologus 155, 394-396.

Kajanto, I., 1965. The Latin Cognomina [= Commentationes Humanarum Litterarum XXXVI.2]. Helsinki: Societas Scientiarum Fennica.

Kaldellis, A., 2004. Procopius of Caesarea. Tyranny, History, and Philosophy at the End of Antiquity. Philadelphia: University of Pennsylvania Press.

Kaldellis, A., 2005. „Republican theory and political dissidence in Ioannes Lydos“. Byzantine and Modern Greek Studies 29, 1-16.

Kaplan, M., 1981. „Novelle de Tibère II sur les «maisons divines »". Centre de recherche d'histoire et civilisation de Byzance. Travaux et mémoires 8, 237-245.

Keenan, J. G., 1973. „The Names Flavius and Aurelius as Status Designations in Later Roman Empire“. Zeitschrift für Papyrologie und Epigraphik 11, 33-63.

Kienast, D., 1996. Römische Kaisertabelle. Grundzüge einer römischen Kaiserchronologie. 2., durchges. und erw. Aufl. Darmstadt: Wissenschaftliche Buchgesellschaft.

Kolb, F., 1987. Diocletian und die Erste Tetrachie. Improvisation oder Experiment in der Organisation monarchischer Herrschaft? Berlin / New York: Walter de Gruyter.

Kruse, M., 2015. „A Justinianic Debate across Genres on the State of the Roman Republic“. In: G. Greatrex, H. Elton (ed.), Shifting Genres in Late Antiquity. Farnham / Burlington: Ashgate, 233-245.

Laniado, A., 2002. Recherches sur les notables municipaux dans l'empire protobyzantin. Paris: Association des amis du centre d'histoire et civilisation de Byzance.

Laniado, A., 2004. „L'onomastique romaine dans le monde protobyzantin : quelques témoignages négligés“. Antiquité tardive 12, 325-345.

Laniado, A., 2012. „Parenté, relations et dévotion : le phénomène de polyonymie chez les dignitaires de l'empire protobyzantin (Ve-VIe siècles)“. In: Ch. Badel, Ch. Settipani (ed.), Les stratégies familiales dans l'antiquité tardive. Actes du colloque organisé par le C.N.R.S. USR 710 «L'Année épigraphique » tenu à la Maison des Sciences de l'Homme les 5-7 février 2009. Paris: de Boccard, 27-55.

Leppin, H., 2011. Justinian. Das christliche Experiment. Stuttgart: Klett-Cotta.

Liddell, H. G., Scott, R., Jones, H. S., 1996. A Greek-English Lexicon. Ninth ed. With a revised supplement 1996. Oxford: Clarendon Press.

Maas, M., 1986. „Roman History and Christian Ideology in Justinianic Reform Legislation“. Dumbarton Oaks Papers 40, 17-31.

Maas, P., 1912. „Metrische Akklamationen der Byzantiner“. In: P. Maas, Kleine Schriften. München: C. H. Beck'sche Verlagsbuchhandlung, 1973, 393-418 [ursprünglich: Byzantinische Zeitschrift 21, 28-51].

Meier, M., 2003a. Das andere Zeitalter Justinians. Kontingenzerfahrung und Kontingenzbewältigung im 6. Jahrhundert n. Chr. 2., unveränderte Aufl. Göttingen: Vandenhoeck \& Ruprecht, 2004.

Meier, M., 2003b. „Die Inszenierung einer Katastrophe: Justinian und der Nika-Aufstand“. Zeitschrift für Papyrologie und Epigraphik 142, 273-300.

Meyer, W., 1881. „Zwei antike Elfenbeintafeln der k. Staats-Bibliothek in München“. Abhandlungen der philosophisch-philologischen Classe der Königlich bayerischen Akademie der Wissenschaften 15, I. Abtheilung, 1-84.

Noethlichs, K. L., 2000. „Quid possit antiquitas nostris legibus abrogare? Politische Propaganda und praktische Politik bei Justinian I. im Lichte der kaiserlichen Gesetzgebung und der antiken Historiographie“. Zeitschrift für antikes Christentum 4, 116-132. 
Noethlichs, K. L., 2001. „Iustinianus (Kaiser)“. In: RAC XIX: 668-763.

Oikonomides, N., 1986. „Le dédoublement de saint Théodore et les villes d'Euchaïta et d'Euchaneia“. Analecta Bollandiana 104, 327-335 = N. Oikonomides, Byzantium from the Ninth Century to the Fourth Crusade. Studies, Texts, Monuments. Hampshire / Brookfield, Vermont: Variorum, Nr. I.

Pfeilschifter, R., 2013. Der Kaiser und Konstantinopel. Kommunikation und Konfliktaustrag in einer spätantiken Metropole. Berlin / Boston: De Gruyter.

PLRE I = The Prosopography of the Later Roman Empire. Volume I. A.D. 260-395. Cambridge: Cambridge University Press, 1971.

PLRE II = The Prosopography of the Later Roman Empire. Volume II. A.D. 395-527. Cambridge [et al.]: Cambridge University Press, 1980.

PLRE III = The Prosopography of the Later Roman Empire. Volume III. A.D. 527-641. Volume IIIA-Volume IIIB. Cambridge [et al.]: Cambridge University Press, 1992.

Prchlík, I., 2015. „The Fiery Eyes of Augustus and the Annales of Nicomachus Flavianus“. Acta Universitatis Carolinae Philologica 2015/2 [= Graecolatina Pragensia 25], 9-20.

Psaltes, S. B., 1913. Grammatik der Byzantinischen Chroniken. Göttingen: Vandenhoeck \& Ruprecht.

RAC XIX = Reallexikon für Antike und Christentum. Band XIX. Itinerarium - Kannibalismus. Stuttgart: Anton Hiersemann, 2001.

Rösch, G., 1978. Onoma basileias. Studien zum offiziellen Gebrauch der Kaisertitel in spätantiker und frühbyzantinischer Zeit. Wien: Verlag der Österreichischen Akademie der Wissenschaften.

Roesler, R., 1873. „Ueber den Zeitpunkt der slavischen Ansiedlung an der unteren Donau“. Sitzungsberichte der kaiserlichen Akademie der Wissenschaften. Philosophisch-historische Classe 73, 77-126.

Rosen, K., 2001a. „Iustinus I (Kaiser)“. In: RAC XIX: 763-778.

Rosen, K., 2001b. „Iustinus II (Kaiser)“. In: RAC XIX: 778-801.

Salomies, O., 1992. Adoptive and Polyonymous Nomenclature in the Roman Empire [= Commentationes Humanarum Litterarum 97]. Helsinki: Societas Scientiarum Fennica.

Salway, B., 1994. „What's in a Name? A Survey of Roman Onomastic Practice from c. 700 B.C. to A.D. 700“. The Journal of Roman Studies 84, 124-145.

Schamp, J., 2006. „Analyse de contenu“. In: Jean le Lydien, Des magistratures de l'état romain. Tome I. 1ère partie-2e partie. Texte établi, traduit et commenté par M. Dubuisson et J. Schamp. Paris: Les Belles Lettres, CCLXXXVII-DCCXXXIX.

Shackleton Bailey, D. R., 1976. Two Studies in Roman Nomenclature. [S.l.]: The American Philological Association.

Signes Codoñer, J., 2003. „Kaiserkritik in Prokops Kriegsgeschichte“. In: D. Brodka, J. Jannik, S. Sprawski (ed.), Freedom and Its Limits in the Ancient World. Proceedings of a colloquium held at the Jagiellonian University Kraków, September 2003 [= Electrum 9]. Kraków: Jagiellonian University Press, 215-229.

Stein, E., 1919. Studien zur Geschichte des byzantinischen Reiches vornehmlich unter den Kaisern Justinus II $u$. Tiberius Constantinus. Stuttgart: J. B. Metzlersche Verlagsbuchhandlung.

Stein, E., 1949. Histoire du Bas-Empire. Tome II. De la disparition de l'Empire d'Occident à la mort de Justinien (476-565). Paris / Bruxelles / Amsterdam: Desclée de Brouwer.

Szidat, J., 2014. „Zu Iustinians dies imperii und zum Problem von Datierung in der Osterzeit. Überlegungen zur antiken Überlieferung, besonders zu Constantinus Porphyrogenitus, De cerimoniis aulae Byzantinae 1, 95“. Byzantinische Zeitschrift 107, 877-891.

Vasiliev, A. A., 1950. Justin the First. An Introduction to the Epoch of Justinian the Great. Cambridge, Mass.: Harvard University Press.

Vatin, C., 1962. „Les empereurs du IVe siècle à Delphes“. Bulletin de correspondance hellénique 86, 229-241.

Walter, Ch., 1999. „Theodore, archetype of the warrior saint“. Revue des études byzantines 57, 163-210.

Whitby, Mi., 1994. „Images for emperors in late antiquity: a search for New Constantine“. In: P. Magdalino (ed.), New Constantines. The Rhythm of Imperial Renewal in Byzantium, 4th-13th Centuries. Papers from the Twenthy-sixth Spring Symposium of Byzantine Studies, St Andrews, March 1992. Aldershot: Variorum, 83-93. 


\title{
IUSTINIANOS - NOVÝ AUGUSTUS? \\ ADOPCE, JMÉNO A PROPAGANDA JEDNOHO BUDOUCÍHO CÍSAŘE
}

V článku je navrženo přehodnocení dokladů týkajících se adopce pozdějšího císaře Iustiniana jeho strýcem Iustinem, kterou zpochybnil Noethlichs (2001). Ačkoli Iustinianovy odkazy na Iustina jako „otce“ nejsou zcela průkazné, epigram Anth. Gr. I, 97 lze považovat za spolehlivou pozitivní indicii. Zatímco „Iustinianos“ je císařovo původní jméno a nemá s adopcí nic společného, jména „Petrus“ a „Sabbatius“, známá z konzulárních diptychů, jsou jmény sekundárními, z nichž první snad bylo vzorem pro následující módu devocionálních cognomina. Nakonec jsou probrána související témata možné Iustinianovy propagandy (jeho připodobňování k Augustovi adoptovanému Caesarem) a její recepce (Prokopiem či Ióannem Lydem).

\author{
Ján Bakyta \\ Karlsuniversität, Prag \\ jan.bakyta@ff.cuni.cz
}

